\title{
Mixed Finite Element Methods for Groundwater Flow in Heterogeneous Aquifers
}

\author{
L. Traverso ${ }^{\mathrm{a}, \mathrm{b}}$, T. N. Phillips ${ }^{\mathrm{a}}$, Y. Yang ${ }^{\mathrm{b}}$ \\ ${ }^{a}$ School of Mathematics, Cardiff University, Cardiff, CF24 4 AG, United Kingdom \\ ${ }^{b}$ School of Earth and Ocean Sciences, Cardiff University, Cardiff, CF10 3AT, United \\ Kingdom
}

\begin{abstract}
This paper is concerned with a comparison of the performance and efficiency of mixed finite element methods for solving single phase fluid flow in porous media. Particular attention is given to the accurate determination of the groundwater fluxes. The linear systems generated by the mixed finite element method (MFEM) are indefinite. Symmetric positive definite linear systems are generated through the introduction of Lagrange multipliers giving rise to the mixed hybrid finite element method (MHFEM). The convergence behaviour of the numerical approximations is investigated over a range of conductivity coefficients from heterogeneous, isotropic and diagonal to discontinuous, anisotropic and full, on both triangular and quadrilateral, structured and distorted meshes. The robustness and efficiency of various preconditioned solvers is investigated in terms of optimality with respect to both mesh size and conductivity coefficient.
\end{abstract}

Keywords: Groundwater flow; conductivity coefficient; mixed finite element method; mixed hybrid finite element method; preconditioners; conjugate gradient; MINRES; AMG

\section{Introduction}

The equations governing single phase porous media flow can be solved either as a single second order partial differential equation for potential (pressure) or as a system of two first order partial differential equations for pressure and velocity. Mixed finite element methods (MFEM) are based on the latter. These methods ensure the continuity of fluxes but at the cost of additional degrees of freedom and a discrete problem with an indefinite matrix.

The importance of accurate approximation of fluxes has been fiercely debated in the groundwater modelling community over the last two decades or so. The central issue concerns the importance of accurate flux computations

Email addresses: traversol@cardiff.ac.uk (L. Traverso), PhillipsTn@cf.ac.uk (T. N. Phillips), YangY6@cardiff.ac.uk (Y. Yang) 
not only when solving the contaminant transport equations but also in determining accurate water balances. In many applications of traditional numerical techniques such as finite elements (FEM), finite differences (FDM) and finite volumes (FVM) to groundwater flow problems, an approximation to the potential is generated first and then the flux is obtained by numerical differentiation in a post-processing stage using Darcy's Law $[1,2,3,4]$. Whilst post-processing techniques for determining the flux might be suitable for problems with relatively homogeneous hydraulic conductivity, they are not appropriate for heterogeneous aquifers $[5,6]$. This is because they are prone to numerical error particularly when the hydraulic conductivity coefficient is discontinuous with large contrasts in different regions of the problem domain.

Mixed finite element methods (MFEM) $[7,8]$ represent an alternative to traditional numerical schemes and are based on the simultaneous approximation of potential and groundwater fluxes. Mixed methods are characterised by the choice of vector basis functions used to approximate the normal components of fluxes across each finite element edge (in 2D) or face (in 3D). Additionally, scalar basis functions, which are element-wise constant, are chosen for the approximation of the potential. Mixed methods possess some important properties, e.g. they are locally conservative and the normal components of the fluxes are continuous across element boundaries.

Groundwater fluxes obtained by mixed methods are generally more accurate than those obtained through Darcian post-processing. Numerical evidence to substantiate this claim has been provided by Durlofsky [9] and Kaasschieter [10], for example. However, the improvement in accuracy is achieved at greater computational expense simply due to the larger number of degrees of freedom in the mixed formulation compared to the standard formulation. In the mixed method, the number of unknowns corresponds to the sum of the number of elements and edges in the finite element mesh whereas as in the standard formulation, the number of unknowns corresponds to the number of elements or nodes (FEM and FDM / FVM , respectively). This drawback of mixed methods was one of the arguments used against their use in the papers of Cordes and Kinzelbach [2], and Srivastava and Brusseau [3].

The mathematical theory underpinning the mixed formulation is mature and well-developed (see $[11,12,7,13,14,15,8]$, for example). Existence and uniqueness of MFEM approximations (Raviart-Thomas $R T_{0}$ or Brezzi-DouglasMarini $\left.B D M_{1}[16]\right)$ is guaranteed for any mesh and full-tensor representation of the diffusion coefficient [17]. Extensive research has been carried out on error and convergence analysis for the lowest order Raviart-Thomas $\left(R T_{0}\right)$ mixed finite element method (see Brezzi and Fortin [8], Arbogast et al. [18], Demlow [19], Radu et al. [20], for example); and on comparing MFEM approximations with finite volume and multi-point flux approximation (MPFA) methods $[21,22,23,24,25,26,27,28,29,30,31]$. The link between mixed formulations and standard finite volume methods is investigated in $[25,32,33,34,27])$. Similarly, the link between MPFA and mixed methods is analysed in Vohralik [35], Klausen and Russell [36], Wheeler and Yotov [37], Younes and Fontaine $[38,39]$. Comparative studies between MFEM and other methods focus on com- 
putational aspects (see Kaasschieter and Huijben [5], Younes et al. [40], Younes and Fontaine [38, 39], for example) as well as the quality of the approximations $[29,17]$.

The discrete linear systems of equations generated using the mixed formulation are indefinite. In the past, this was considered to be a considerable weakness of the approach due to the lack of an efficient iterative scheme for solving saddlepoint problems. However, this deficiency can be circumvented by augmenting the linear system using Lagrange multipliers, thereby creating what is known as the mixed-hybrid finite element method (MHFEM) [7, 8]. The discrete linear systems obtained by MHFEM are symmetric positive definite (SPD) and therefore can be efficiently solved using the conjugate gradient (CG) method. Additionally, the size of the linear system is reduced (to one of dimension equal to the number of edges) by eliminating the pressure and velocity unknowns from the system. Hence, based solely on the size of the discrete linear system, MHFEM is computationally less expensive than classical MFEM. Consequently, in the majority of the comparative studies with MPFA and finite volume methods cited above, the SPD version is used. Studies associated with the classical MFEM are significantly less common in the literature as this represents a somewhat specialised area of research. Note, however, that the saddle-point problem obtained from the mixed formulation can be solved using the minimal residual (MINRES) method [41]. If MINRES is preconditioned with efficient symmetric preconditioners then the solution of the symmetric indefinite system can be very efficient $[42,43,44,45,46]$. The choice of preconditioner for MINRES is crucial to its competitiveness.

This paper focuses on MFEM both in its classical and hybrid formulations. It investigates MFEM convergence performance for a range of conductivity coefficients often encountered in groundwater modelling applications. These range from heterogeneous, isotropic and diagonal to discontinuous, anisotropic and full tensor coefficients. We show that accurate approximations for the flux and potential unknowns are achieved in all settings. We highlight the cases where loss of convergence accuracy is experienced on triangular and quadrilateral, structured and distorted meshes.

In addition to the error analysis the paper reports the computational cost of solving the indefinite linear systems derived using MFEM (Raviart-Thomas elements of lowest order) and the symmetric positive definite systems obtained with hybridization. Throughout the paper the focus is on robustness of the solvers and preconditioners with respect to the conductivity coefficient and the discretisation parameter of the numerical scheme. We provide further evidence to show that if MINRES is equipped with an efficient preconditioner such as the one proposed by Powell and Silvester [45], solving the indefinite system can be more efficient than solving the SPD case.

The numerical experiments that have been conducted and described in this paper encompass a wide range of conductivity coefficients and different meshes. Consequently, the paper provides a complete comparative study on computational cost of the classical and hybrid mixed finite element methods for solving groundwater flow problems. 


\section{Mathematical Model}

The steady-state flow of water in porous media is described by a scalar second-order partial differential equation, the solution of which, when supplemented with suitable boundary conditions, gives the distribution of a scalar potential $u$ (potential head). Let $D$ be a domain in $\mathbb{R}^{d}, d=2,3$, with Lipschitz continuous boundary $\Gamma$. Let $\Gamma=\Gamma_{D} \cup \Gamma_{N}$ with $\Gamma_{D} \cap \Gamma_{N}=\emptyset$, where $\Gamma_{D}$ and $\Gamma_{N}$ denote the portions of $\Gamma$ where Dirichlet and Neumann boundary conditions on $u$ are prescribed, respectively.

We seek a solution $u$ to the second-order elliptic problem

$$
\begin{aligned}
-\nabla \cdot \mathcal{C} \nabla u & =f & & \text { in } D, \\
u & =g & & \text { on } \Gamma_{D}, \\
\mathcal{C} \nabla u \cdot \mathbf{n} & =0 & & \text { on } \Gamma_{N},
\end{aligned}
$$

where $\mathcal{C}$ is a given $d \times d$ symmetric positive definite coefficient tensor representing the hydraulic conductivity, $\mathbf{n}$ denotes the unit outward normal vector to $\Gamma_{N}, g$ represents the prescribed constant head on $\Gamma_{D}$ and $f$ represents a sink or source term.

Traditionally, finite difference or finite element methods have been used to discretise problem (1). In such methods it is common to post-process the approximation to $u$ in order to obtain the fluid discharge (flux) or velocity, q, according to Darcy's Law. Whilst this is commonly done in practice, many authors have shown that the computed fluxes are inaccurate due to errors introduced by numerical differentiation (see [5] and [6], for example).

In many applications, $\mathbf{q}$ is the primary variable of interest. Hence, a numerical scheme which guarantees an accurate approximation of the fluxes is preferred. This can be achieved by recasting problem (1) as a first-order system of partial differential equations in which Darcy's Law appears explicitly. We now seek the solution $(\mathbf{q}, u)$ to the coupled first-order problem

$$
\begin{aligned}
\mathcal{C}^{-1} \mathbf{q}-\nabla u & =0 & & \text { in } D, \\
\nabla \cdot \mathbf{q} & =-f & & \text { in } D, \\
u & =g & & \text { on } \Gamma_{D}, \\
\mathbf{q} \cdot \mathbf{n} & =0 & & \text { on } \Gamma_{N} .
\end{aligned}
$$

The solution of problem (2) using mixed finite element methods allows us to obtain, simultaneously, approximations for $u$ and $\mathbf{q}$ everywhere in $D$.

\section{Weak Formulation}

In this section we introduce the weak formulation of the problem. The mixed finite element method is based on a discretization of the weak formulation. The functions vanishing on $\Gamma_{D}$ belong to the Hilbert space

$$
H_{0, D}^{1}(D)=\left\{w \in H^{1}(D): w=0 \text { on } \Gamma_{D}\right\} .
$$


The following Hilbert spaces are required for the mixed variational formulation of problem (2). The space $H(d i v ; D)$ is defined by

$$
H(\operatorname{div} ; D)=\left\{\mathbf{v}: \mathbf{v} \in L^{2}(D)^{d} \text { and } \nabla \cdot \mathbf{v} \in L^{2}(D)\right\},
$$

equipped with the inner product

$$
(\mathbf{v}, \mathbf{u})_{d i v}=(\mathbf{v}, \mathbf{u})+(\nabla \cdot \mathbf{v}, \nabla \cdot \mathbf{u}),
$$

and associated norm

$$
\|\mathbf{v}\|_{d i v}=\|\mathbf{v}\|_{L^{2}(D)^{d}}+\|\nabla \cdot \mathbf{v}\|_{L^{2}(D)} .
$$

Define $w_{\Gamma}$ to be the trace of any scalar function $w \in H^{1}(D)$. The set of all such traces defines the Hilbert space

$$
H^{\frac{1}{2}}(\Gamma)=\left\{g: g=w_{\Gamma} \text { for some } w \in H^{1}(D)\right\} .
$$

Similarly, for vector functions $\mathbf{v} \in H(\operatorname{div} ; D),(\mathbf{v} \cdot \mathbf{n})_{\Gamma}$ defines the normal trace, where $\mathbf{n}$ is the unit outward normal to $\Gamma$. Therefore the set of all such functions determines

$$
H^{-\frac{1}{2}}(\Gamma)=\left\{q: q=(\mathbf{v} \cdot \mathbf{n})_{\Gamma} \text { for some } \mathbf{v} \in H(\operatorname{div} ; D)\right\} .
$$

Following Powell [44], for any function $g \in H^{\frac{1}{2}}(\Gamma)$ and $q \in H^{-\frac{1}{2}}(\Gamma),\langle\cdot, \cdot\rangle$ represents the duality pairing

$$
\langle g, q\rangle=\int_{\Gamma} g q d s,
$$

and we define the subspace of $H(\operatorname{div} ; D)$ in which the solution is sought

$$
H_{0, N}(\operatorname{div} ; D)=\left\{\mathbf{v} \in H(\operatorname{div} ; D):\langle\mathbf{v} \cdot \mathbf{n}, w\rangle=0 \quad \forall w \in H_{0, D}^{1}(D)\right\} .
$$

Define $W=L^{2}(D)$ and $V=H(\operatorname{div} ; D)$. The weak formulation of the mixed variational problem (2) is: Find $(\mathbf{q}, u) \in V \times W$ such that

$$
\begin{aligned}
a(\mathbf{q}, \mathbf{v})+b(\mathbf{v}, u) & =\langle g, \mathbf{n} \cdot \mathbf{v}\rangle_{\Gamma_{D}} & & \forall \mathbf{v} \in V \\
b(\mathbf{q}, w) & =-(f, w) & & \forall w \in W
\end{aligned}
$$

where the bilinear forms $a(\cdot, \cdot)$ and $b(\cdot, \cdot)$ are defined by

$$
\begin{aligned}
a(\mathbf{q}, \mathbf{v}) & =\int_{D} \mathcal{C}^{-1}(\mathbf{q} \cdot \mathbf{v}) d \Omega \\
b(\mathbf{v}, w) & =\int_{D} \nabla \cdot \mathbf{v} w d \Omega,
\end{aligned}
$$

respectively. 
The weak formulation has a unique solution $(\mathbf{q}, u) \in V \times W$ provided the bilinear forms $a(\cdot, \cdot)$ and $b(\cdot, \cdot)$ satisfy the following inf-sup condition (also called the Ladyzhenskaya-Babuška-Brezzi (LBB) condition)

$$
\inf _{w \in W} \sup _{\mathbf{v} \in V} \frac{\int_{D} w \nabla \cdot \mathbf{v}}{\|w\|_{W}\|\mathbf{v}\|_{V}} \geq \beta,
$$

where the constant $\beta>0$ (for a proof of this condition see Brezzi and Fortin $[8])$.

\section{Mixed Finite Element Method}

Let $T^{h}$ be a partition of $D$ defined by closed sub-domains (finite elements) $K_{i}, i=1, \ldots, n$, such that,

$$
T^{h}=\bigcup_{i=1}^{n} K_{i}
$$

where $h$ denotes the discretisation parameter which describes the size of the finite elements in $T^{h}$. Let $E^{h}$ be the collection of numbered edges $(d=2)$ or faces $(d=3), e_{i}, i=1, \ldots, m$, where $m$ is the total number of edges or faces in $T^{h}$. According to the Galerkin method we define finite dimensional subspaces $V^{h} \subset V$ and $W^{h} \subset W$. The discrete variational formulation of (10) is: Find $\left(\mathbf{q}^{h}, u^{h}\right) \in V^{h} \times W^{h}$ such that

$$
\begin{aligned}
a\left(\mathbf{q}^{h}, \mathbf{v}^{h}\right)+b\left(\mathbf{v}^{h}, u^{h}\right) & =\left\langle g, \mathbf{n} \cdot \mathbf{v}^{h}\right\rangle_{\Gamma_{D}} & & \forall \mathbf{v}^{h} \in V^{h} \\
b\left(\mathbf{q}^{h}, w^{h}\right) & =-\left(f, w^{h}\right) & & \forall w^{h} \in W^{h}
\end{aligned}
$$

\subsection{Raviart-Thomas Approximation}

A family of local spaces that can be used to construct a suitable subspace $V^{h} \subset V \equiv H_{0, N}($ div $; \Omega)$ was proposed by Raviart and Thomas [11] for $\mathbb{R}^{2}$ and by Nedelec [12] for $\mathbb{R}^{3}$. Let $R T^{0}$ denote the space of linear vector functions $\mathbf{v}_{i}$, $i=1, \ldots, I$, where $I$ is the number of edges or faces associated with a finite element $K$. Therefore, we have

$$
R T^{0}(K)=\operatorname{span}\left\{\mathbf{v}_{i}\right\}_{i=1}^{I} .
$$

The value of $I$ depends on the type of finite element chosen for the discretisation of $D$, so that $I=3$ and $I=4$ for triangular and rectangular elements, respectively, and $I=4$ and $I=6$ for tetrahedra and parallelepipeda, respectively.

It is common practice to define the vector basis functions on a reference element $\hat{K}$. Thus the definition of vector basis functions on a general element follows from the reference element through an affine transformation. In such circumstances the well-known transformation rules for vector and scalar basis functions apply (see Brezzi and Fortin [8], §III.1.3). Let $R T^{0}(\hat{K})$ denote the 
local $I$-dimensional space of vector basis functions $\hat{\mathbf{v}}_{i}$ defined on $\hat{K}$. It follows that

$$
R T^{0}(K)=\left\{\mathbf{v}: \mathbf{v}(\mathbf{x})=\frac{\mathbf{B} \hat{\mathbf{v}}(\boldsymbol{\xi})}{J} \forall \boldsymbol{\xi} \in \hat{K} \text { and } \hat{\mathbf{v}} \in R T^{0}(\hat{K})\right\},
$$

where $\boldsymbol{\xi}$ is the local coordinate system and $J$ is the determinant of the Jacobian of the transformation $\mathbf{B}$. We can now define the global spaces

$$
R T^{0}\left(D ; T^{h}\right)=\left\{\mathbf{v} \in H(\operatorname{div} ; D):\left.\mathbf{v}\right|_{K} \in R T^{0}(K) \forall K \in T^{h}\right\},
$$

and

$$
\mathcal{M}^{0}=\left\{\mathbf{v} \in L^{2}(D)^{d} \text { and }\left.\mathbf{v}\right|_{K} \in R T^{0}(K) \forall K \in T^{h}\right\} .
$$

A suitable subspace for the approximation to the flux $\mathbf{q}$ is

$$
V^{h}=\mathcal{M}^{0} \cap H_{0, N}(\operatorname{div} ; D)=\left\{\mathbf{v} \in R T^{0}\left(D ; T^{h}\right) \text { and }\left.\mathbf{v} \cdot \mathbf{n}\right|_{\Gamma_{N}}=0\right\} .
$$

For triangular and tetrahedra elements the vector basis functions $\hat{\mathbf{v}} \in R T^{0}(\hat{K})$ have the special form

$$
\hat{\mathbf{v}}=\left(\begin{array}{c}
a+c \xi \\
b+c \eta
\end{array}\right), \quad \hat{\mathbf{v}}=\left(\begin{array}{c}
a+c \xi \\
b+c \eta \\
e+c \zeta
\end{array}\right),
$$

respectively, and for rectangular and parallelepipeda elements they take the form

$$
\hat{\mathbf{v}}=\left(\begin{array}{c}
a+c \xi \\
b+d \eta
\end{array}\right), \quad \hat{\mathbf{v}}=\left(\begin{array}{c}
a+c \xi \\
b+d \eta \\
e+f \zeta
\end{array}\right) .
$$

respectively. The coefficients $a, b, c, d, e$, and $f$ are constants chosen so that the integral of the normal component of $\hat{\mathbf{v}}$ along an edge or face of $\hat{K}$ is equal to some constant $\delta$.

Finally, the potential $u$ is approximated by piecewise constant functions $w$. Let $M^{0}(K)$ denote the one-dimensional space of constant scalar basis functions on $K$. Hence, a suitable subspace $W^{h} \subset W \equiv L^{2}(D)$ is

$$
W^{h}=\left\{w \in L^{2}(D):\left.w\right|_{K} \in M^{0}(K) \forall K \in T^{h}\right\} .
$$

\subsection{Linear System}

For each element $K$ we associate a scalar basis function $\phi_{j}, j=1, \ldots, n$, which is element-wise constant. The potential $u^{h}$ can therefore be approximated in terms of the global scalar basis functions,

$$
u^{h}=\sum_{j=1}^{n} u_{j} \phi_{j},
$$


where $\phi_{j}$ is the characteristic function on $K_{j}$. Globally, for each edge or face $e \in E^{h}$ we fix oriented normal vectors $\boldsymbol{\nu}_{i}, i=1, \ldots, m$, where $m$ is the total number of edges in $E^{h}$. Next, we define a direction index $s_{K}^{i}$ so that

$$
s_{K}^{i}= \begin{cases}+1 & \text { if } \mathbf{n}_{K}^{i}=\boldsymbol{\nu}_{K}^{i} \\ -1 & \text { if } \mathbf{n}_{K}^{i}=-\boldsymbol{\nu}_{K}^{i}\end{cases}
$$

where $\mathbf{n}_{K}^{i}$ denotes the set of unit outward normal vectors to the edges $e_{i} \in E^{h}$.

The vector (flux) basis functions $\hat{\varphi}_{i} \in V^{h}$ are defined with respect to a reference element $\hat{K}$ so that,

$$
\int_{e_{k}} \hat{\boldsymbol{\varphi}}_{i} \cdot \hat{\boldsymbol{\nu}}_{k} d s=\left\{\begin{array}{ll}
1 & \text { if } k=i \\
0 & \text { if } k \neq i
\end{array} .\right.
$$

Note that this is the condition which ensures continuity of the normal components of the flux $\mathbf{q}$ across the inter-element edges of $E^{h}$. Finally, we can approximate $\mathbf{q}^{h}$ in terms of the global vector basis functions $\varphi_{i}$,

$$
\mathbf{q}^{h}=\sum_{i=1}^{m} q_{i} \boldsymbol{\varphi}_{i}
$$

The mapping $\hat{\boldsymbol{\varphi}}_{i} \mapsto \boldsymbol{\varphi}_{i}$ follows from (13). Additionally, the global basis functions $\varphi_{i}$ are multiplied by the index $s_{i}$ before the system is assembled. The source / sink term $f$ is also approximated in terms of the global scalar basis functions $\phi_{i}$

$$
f \approx \sum_{i=1}^{n} f_{i} \phi_{i}
$$

Substituting expansions (18), (21) and (22) into (12) we obtain

$$
\begin{aligned}
\sum_{j=1}^{m} A_{i, j} q_{j}+\sum_{k=1}^{n} B_{i, k} u_{k} & =g_{i} \quad i=1, \ldots, m, \\
\sum_{i=1}^{m} B_{k, i} q_{i} & =f_{k} \quad k=1, \ldots, n,
\end{aligned}
$$

where $A_{i, j}$ is constructed from the element contributions

$$
A_{i, j}^{K}=a\left(\boldsymbol{\varphi}_{j}, \boldsymbol{\varphi}_{i}\right)_{K}=\int_{K} \mathcal{C}^{-1} \boldsymbol{\varphi}_{j}^{K} \boldsymbol{\varphi}_{i}^{K} d K, \quad i, j=1, \ldots, I,
$$

where $I$ is the number of edges or faces on $K$. Given an element $K, \phi_{K}=1$, hence the element contributions to the global matrix $B_{k, i}$ are given by

$$
B_{i}^{K}=\int_{e_{i}} \boldsymbol{\varphi}_{i} \cdot \mathbf{n}_{i} d e, \quad i=1, \ldots, I
$$


and

$$
B_{k, i}=\left\{\begin{array}{ll}
0 & \text { if } e_{i} \notin K_{k} \\
s_{i}^{K_{k}} & \text { if } e_{i} \in K_{k}
\end{array} .\right.
$$

The elements of the right-hand side vectors are defined by

$$
f_{k}=\int_{K_{k}} f d K_{k} \quad g_{i}=\left\{\begin{array}{ll}
0 & \text { if } e_{i} \notin \Gamma_{D} \\
\int_{e_{i}} g d e & \text { if } e_{i} \in \Gamma_{D}
\end{array} .\right.
$$

The system (23) can be re-written in matrix notation as follows

$$
\left(\begin{array}{cc}
A & B^{T} \\
B & 0
\end{array}\right)\left(\begin{array}{l}
\boldsymbol{q} \\
\boldsymbol{u}
\end{array}\right)=\left(\begin{array}{l}
\boldsymbol{g} \\
\boldsymbol{f}
\end{array}\right),
$$

where $\boldsymbol{q}=\left[q_{1}, \ldots, q_{m}\right]^{T}$ and $\boldsymbol{u}=\left[u_{1}, \ldots, u_{n}\right]^{T}$. The symmetric matrix $A$ is generally referred to as a weighted velocity matrix and the matrix $B$ is a discrete representation of the divergence operator.

Given that $A$ is positive definite, we can write

$$
\mathbf{q}=A^{-1}\left(\mathbf{g}-B^{T} \mathbf{u}\right),
$$

which when substituted into the second equation of (28) gives

$$
B A^{-1} B^{T} \mathbf{u}=B A^{-1} \mathbf{g}-\mathbf{f} .
$$

The matrix $B A^{-1} B^{T}$ is also symmetric and positive definite (see Chavent and Jaffré [13] and Kaasschieter and Huijben [5] for an alternative proof). These properties are very important with regard to the choice of method used to solve the linear system (28).

\subsection{Solution Strategies}

A review of solution strategies for the linear system (28) can be found in the theses of Scheichl [47] and Powell [44]. The solution of system (30) by the conjugate gradient method is advocated by Kim [48] and Ewing and Wheeler [49]. However, the computation of $A^{-1}$ is expensive for general meshes and for full-tensor $\mathcal{C}$ and the Schur complement $B A^{-1} B^{T}$ is not sparse. When rectangular meshes and diagonal conductivity coefficients $\mathcal{C}$ are used the elemental contributions $A_{K}$ to the weighted velocity matrix are block-diagonal (see [44]). Hence, the computation of $A_{K}^{-1}$ is inexpensive and the system (30) can be solved efficiently using CG. Additionally, it can be shown that if the trapezoidal rule is used to approximate the elemental contributions to $A$ and $B$ (Kaasschieter and Huijben [5]) on rectangular meshes, then $A$ becomes diagonal. For these special cases the solution of (30) using CG is recommended.

There has been several attempts to solve the saddle-point problem (28) using iterative methods. The Uzawa method is an established iterative scheme for solving saddle-point systems. However, this method requires the computation of 
the inverse of the coefficient matrix which becomes infeasible for practical applications (unstructured meshes and full-tensor coefficients). Fortin and Glowinski [50] introduced the augmented Lagrangian method which applies an Uzawa algorithm to a modified saddle-point problem.

Algebraic approaches to solving (28) were introduced by Rusten and Wither [42] and several preconditioners are proposed in Rusten and Wither [51] and Rusten et al. [52]. Powell [44] and Powell and Silvester [45] proposed an ideal and practical preconditioner of the form

$$
P=\left(\begin{array}{cc}
\operatorname{diag}(A) & 0 \\
0 & B \operatorname{diag}(A)^{-1} B^{T}
\end{array}\right) .
$$

The Schur complement $B \operatorname{diag}(A)^{-1} B^{T}$ can be inverted exactly or approximated using one V-cycle of black-box Algebraic Multi-Grid (AMG).

A preconditioner is defined to be $h$-optimal if the number of iterations required to solve the preconditioned system is independent or almost independent of the discretisation parameter $h$. Powell and Silvester [45] showed that the preconditioner defined by (31) is $h$-optimal for isotropic $\mathcal{C}$ on structured triangular and rectangular meshes. However $h$-optimality is lost for diagonal anisotropic coefficients on triangular meshes. Furthermore, the preconditioner (31) is never $h$-optimal for general full-tensor coefficients.

The definition of $\mathcal{C}$-optimality follows from above. The preconditioner (31) is only $\mathcal{C}$-optimal in some special cases. In fact, its efficiency decreases drastically for anisotropic diagonal and full tensor coefficients on structured triangular meshes. For structured rectangular meshes the preconditioner (31) is more efficient displaying $\mathcal{C}$ optimality for anisotropic diagonal coefficients. Currently, a preconditioner for $(28)$ which is $\mathcal{C}$-optimal for anisotropic full tensor coefficients remains elusive. Furthermore, the efficiency of the preconditioner (31) has not yet been investigated for unstructured two-dimensional meshes and structured and unstructured three-dimensional meshes.

An alternative approach which has been advocated in the literature [10] is the hybrid method, introduced by de Veubeke [53] and further developed by Arnold and Brezzi [7] and Brezzi and Fortin [8]. This is discussed further in the next section.

\section{Mixed Hybrid Finite Element Method}

Arnold and Brezzi [7] presented an alternative discretization of the problem that results in the generation of a symmetric positive definite coefficient matrix for problem (2). The continuity condition on the normal components of the flux $\mathbf{q}$ across the finite element edges or faces is relaxed, i.e. $\mathbf{q}$ is allowed to be discontinuous across element interfaces. The continuity condition (required for the type of problems herein investigated) is subsequently re-established through the introduction of Lagrange multipliers $\lambda$ associated with those interfaces. Since the velocity is discontinuous across element boundaries, the velocity unknowns can be eliminated obtaining from the system to obtain a reduced system with 
unknowns comprising $u^{h}$ and the Lagrange multipliers $\lambda^{h}$. Note that the Lagrange multipliers can be interpreted as approximations to the potential $u$ at the element interfaces. Furthermore the unknowns $u^{h}$ can also be eliminated to obtain a system of equations solely in terms of the Lagrange multipliers $\lambda^{h}$. This final reduced system is positive-definite and of size $m \times m$, where $m$ is the number of edges or faces in $T^{h}$. Hence the conjugate gradient can be used to solve the linear system efficiently.

In the following discussion we use the notation of Brezzi and Fortin [8]. Let $\Lambda_{0}(e)$ denote the space of constant functions on $e, \forall e \in E^{h}$. We define the multiplier space

$$
\Lambda_{0}\left(E^{h}\right)=\left\{\lambda^{h}:\left.\lambda^{h}\right|_{e} \in \Lambda_{0}(e) \forall e \in E^{h}\right\},
$$

and the subspaces of multipliers that either vanish or satisfy the essential boundary condition $u=g$ on $\Gamma_{D}$

$$
\begin{aligned}
& \Lambda_{0, \Gamma_{D}}=\left\{\lambda \in \Lambda\left(E_{h}\right): \lambda=0 \text { on } \Gamma_{D}\right\} \\
& \Lambda_{g, \Gamma_{D}}=\left\{\lambda \in \Lambda\left(E_{h}\right): \lambda=g^{h} \text { on } \Gamma_{D}\right\}
\end{aligned}
$$

where

$$
\int_{e}\left(g^{h}-g\right) d s=0, \quad \forall e \in \Gamma_{D} .
$$

The flux approximation $\mathbf{q}^{h}$ is now sought in $\mathcal{M}^{0}$ and the Lagrange multipliers are defined in $\Lambda_{0}(e)$. Hence the following bilinear forms are defined

$$
\begin{aligned}
c\left(\mu^{h}, \mathbf{q}^{h}\right) & =\sum_{K \in T^{h}} \int_{\Gamma_{K}} \mu^{h} \mathbf{q}^{h} \cdot \mathbf{n} d \Gamma_{K} \\
b\left(\mathbf{q}^{h}, w^{h}\right)_{h} & =\sum_{K \in T^{h}} \int_{K}\left(\nabla \cdot \mathbf{q}^{h}\right) w^{h} d K
\end{aligned}
$$

The hybrid version of the lowest-order Raviart-Thomas mixed method for problem (2) reads: Find $\left(\mathbf{q}^{h}, u^{h}, \lambda^{h}\right) \in \mathcal{M}^{0} \times W^{h} \times \Lambda_{g, \Gamma_{D}}$ such that

$$
\begin{aligned}
a\left(\mathbf{q}^{h}, \mathbf{v}^{h}\right)+b\left(\mathbf{v}^{h}, u^{h}\right)_{h} & =c\left(\lambda_{h}, \mathbf{v}^{h}\right), & & \forall \mathbf{v}^{h} \in \mathcal{M}^{0} \\
b\left(\mathbf{q}^{h}, w^{h}\right)_{h} & =-\left(f, w^{h}\right), & & \forall w^{h} \in W^{h} \\
c\left(\mu^{h}, \mathbf{q}^{h}\right) & =0, & & \forall \mu^{h} \in \Lambda_{0, \Gamma_{D}} .
\end{aligned}
$$

Given the space $\mathcal{M}^{0}$ as defined in (15) and the vector basis functions defined in $\S 4.1$, the approximation for the flux, $\mathbf{q}^{h}$, can be expressed as follows

$$
\mathbf{q}^{h}=\sum_{K \in T^{h}} \sum_{i=1}^{I^{K}} q_{i}^{K} \boldsymbol{\varphi}_{i}^{K},
$$

where $I=3,4,6$, depending on the choice of finite elements for the discretisation of $T^{h}$. The potential $u$ is approximated as in (18). 
Let $\mathcal{I}^{h}=\left\{e \in E^{h}: e \not \subset \Gamma_{D}\right\}$ be the collection of edges $(\mathcal{D}=2)$ or faces $(\mathcal{D}=3) e_{i}, i=1, \ldots, l$, of the finite element mesh excluding those on $\Gamma_{D}$. The space $\Lambda_{0, \Gamma_{D}}$ is spanned by scalar basis functions $\chi_{i}, i=1, \ldots, l$, that satisfy the following condition

$$
\chi_{i}=\left\{\begin{array}{ll}
1 & \text { if } e_{i} \in \mathcal{I}^{h} \\
0 & \text { elsewhere }
\end{array} .\right.
$$

The approximation of the Lagrange multipliers, $\lambda^{h}$, can now be stated as follows

$$
\lambda^{h}=\sum_{i=1}^{l} \lambda_{i} \mu_{i} .
$$

Problem (35) can be expressed in matrix notation as follows

$$
\left(\begin{array}{ccc}
A & B^{T} & C^{T} \\
B & 0 & 0 \\
C & 0 & 0
\end{array}\right)\left(\begin{array}{c}
\mathbf{q} \\
\mathbf{u} \\
\boldsymbol{\lambda}
\end{array}\right)=\left(\begin{array}{c}
\mathbf{g} \\
\mathbf{f} \\
\mathbf{0}
\end{array}\right) .
$$

The clear distinction between (39) and (12) is the choice of the approximation space for the flux $\mathbf{q}$. The space $\mathcal{M}^{0}$ does not require the continuity of $\mathbf{q}^{h} \cdot \mathbf{n}$ which characterizes the space $V^{h}$ and in a more general sense the spaces $H(\operatorname{div} ; D)$. The basis for $\mathcal{M}^{0}$ is chosen so that $\left.\mathbf{q}^{h}\right|_{K} \neq 0$ only in $K$ and vanishes elsewhere. The important advantage of defining $\mathbf{q}^{h}$ in a discontinuous space is that the matrix $A$ becomes block-diagonal and $\mathbf{q}$ can be eliminated at the element level as follows

$$
\mathbf{q}=A^{-1}\left(\mathbf{g}-B^{T} \mathbf{u}-C^{T} \boldsymbol{\lambda}\right)
$$

Note that, inverting $A$ entails inverting its diagonal blocks. Thus this operation can be performed at the element level with little computational expense. Now, using (40) to eliminate $\mathbf{q}$ from (39) we obtain the following reduced system

$$
\left(\begin{array}{cc}
B A^{-1} B^{T} & B A^{-1} C^{T} \\
C A^{-1} B^{T} & C A^{-1} C^{T}
\end{array}\right)\left(\begin{array}{c}
\mathbf{u} \\
\boldsymbol{\lambda}
\end{array}\right)=\left(\begin{array}{c}
B A^{-1} \mathbf{g}-\mathbf{f} \\
C A^{-1} \mathbf{g}
\end{array}\right) .
$$

The matrix $B A^{-1} B^{T}$ is symmetric and positive definite (see [8] and [5] for the proof) and also diagonal [5]. Therefore, we can eliminate $\mathbf{u}$ to obtain

$$
\mathbf{u}=\left(B A^{-1} B^{T}\right)^{-1}\left(B A^{-1} \mathbf{g}-B A^{-1} C^{T} \boldsymbol{\lambda}-\mathbf{f}\right) .
$$

Finally, using (42) to eliminate $\mathbf{u}$ in (41) we obtain the linear system

$$
D \boldsymbol{\lambda}=\mathbf{r},
$$

where

$$
D=C A^{-1} C^{T}-C A^{-1} B^{T}\left(B A^{-1} B^{T}\right)^{-1} B A^{-1} C^{T}
$$

and

$$
\mathbf{r}=C A^{-1} \mathbf{g}+C A^{-1} B^{T}\left(B A^{-1} B^{T}\right)^{-1}\left(\mathbf{f}-B A^{-1} \mathbf{g}\right) .
$$

The matrix $D$ is symmetric and positive definite, hence (43) can be solved using the conjugate gradient method. 


\subsection{Solution Strategies}

As already noted the linear system (43) can be solved using the CG solver. There is a vast number of choices of preconditioner based on $D$ that can be used in conjunction with CG such as SSOR or an incomplete LU (ILU) factorisation of $D$ (see Saad [54]).

A performance analysis for (43) using an incomplete Choleski factorization of $D$ is available in Kaasschieter and Huijben [5]. Several authors use CG for (43) equipped with various preconditioners. Younes and Fontaine [38] use the efficient CG implementation of Eisenstat [55]. The numerical experiments reported exhibit neither $h$-optimality nor $\mathcal{C}$-optimality. To our knowledge, an efficient preconditioner for (43) is currently not available.

Multigrid methods for symmetric and positive definite systems have been extensively studied, and theory, implementation and applications are available in standard reference books (see Briggs et al. [56], Hackbusch [57], for example). Convergence results for multigrid methods for nonconforming finite elements are given in Brenner [58, 59] and Braess and Verfürth [60]. Further results and comparison with mixed methods are given in Chen [61]. Although numerical results presented in these papers show $h$-optimality, analysis of the effect of $\mathcal{C}$ is not included. The effect of the conductivity coefficient on the convergence of AMG is considered by Powell [44]. However, results for unstructured and 3D meshes are not provided.

In this work, we follow the ideas presented in Powell [44] approximating $D$ by one V-cycle of AMG as preconditioner for (43). We extend the implementation to distorted meshes.

The efficient solution of problems (1) and (2) for full-tensor, highly anisotropic coefficients remains a very active research field. Some authors have used sparse direct solvers for this purpose. Recently Younes and Fontaine [39] demonstrated the efficiency of sparse direct solvers based on unifrontal/multifrontal methods $[62,63]$ to solve $(43)$ on quadrilateral meshes. Comparison with iterative methods is not provided.

The efficiency of sparse direct solvers such as UMFPACK [64] depends on the size of the problem. The general consensus is that sparse direct solvers are very efficient for $2 \mathrm{D}$ problems, but their performance deteriorates in 3D. Certainly the point at which sparse direct solvers become less efficient than iterative solvers is problem dependent. In this paper only experiments using iterative solvers are reported.

\section{Numerical Experiments}

In this section the computational cost required to solve the linear systems of equations derived from the MFE and MHFE discretisations is evaluated. Stateof-the-art iterative solvers equipped with efficient preconditioners are used to

solve these systems. The computational cost is evaluated based on number of iterations $N_{i t}$, required by the solver to achieve convergence, and the CPU time $t_{C P U}$ in seconds. 
The codes have been developed within the MATLAB environment [65] and the computations are all performed in serial. The development of the same algorithms in a parallel architecture is a subject for future work and development. The implementation of the Preconditioned Conjugate Gradient (PCG) algorithm follows Saad [54] and the MINRES implementation was modified from Fischer [66]. The tolerance within the solvers is set to $10^{-10}$ and the maximum number of iterations is set to maxit $=10^{4}$. All numerical experiments have been carried out using a standard dual-core laptop computer with 4GB of RAM.

The primary aim of this section is to investigate the circumstances under which the solution of the hybrid problem becomes more efficient than the solution of the indefinite system generated by the mixed method. As expected, there is not a simple universal answer to this question and we show that the relative efficiency of the two approaches is strictly dependent on the on the nature of the problem being considered.

Therefore, several test problems, differing in the form of the conductivity coefficient $\mathcal{C}$, will be analysed. Numerical simulations are performed on both structured and distorted triangular and rectangular meshes to assess the effect of the discretisation on the performance of the solvers. Throughout the discussion, emphasis is given to those situations where $h$ and $\mathcal{C}$ optimality is achieved.

Two tables are presented for each test problem. The first table includes results for preconditioned MINRES using (31) with a direct solver for the Schur complement. The preconditioned CG solver is used for the MHFE formulation (39) using an incomplete Choleski factorisation of the matrix $D$ as preconditioner. These solvers are referred to as $p$-MINRES and PCG in the tables and following subsections, respectively. In the second table results are presented for MINRES with one V-cycle of black-box AMG used for the approximation of the Schur complement. The preconditioner for CG is the AMG approximation of the coefficient matrix $D$. These solvers are referred to as $p$-MINRES $A M G$ and $\mathrm{PCG}_{A M G}$ in the tables and following sections, respectively.

The AMG solver we use is publicly available from the PIFISS [67] library and is written in MATLAB. Other versions written in FORTRAN / MATLAB such as the HSL_MI20 $[68,69]$ are also freely available for academic use. Two types of smoothing functions are available in the library, these are the point GaussSeidel (PGS) and the point damped Jacobi (PDJ). In the following experiments we use the latter with two sweeps per iteration. Note that there is no attempt at tuning the several AMG parameters and that experiments with PGS were not performed.

Note that the setup time for some of the preconditioners can be significantly large especially for fine meshes. Accordingly, the setup time as well as the solution time are reported in the tables. Whenever the set-up time is not negligible this is reported in the tables separately in the following manner: "set-up time" + "solution time".

\subsection{Triangular Meshes}

We restrict ourselves to the case in which $D$ is a square domain. Structured meshes are obtained by partition of $D$ into regular squares of area $h^{2}$. Each 
square is further subdivided into two right angled triangles to obtain a total of $N_{e}$ finite elements. Distorted meshes are created by perturbation of structured meshes as explained later.

The analytical and numerical solutions for each test problem are presented. However, given that the MFEM and MHFEM approximations are equivalent, we only present results for the former method. Similarly, only the $L^{2}$-norms of the error in the potential and velocity approximations for the MFEM are tabulated. The $L^{2}$-norms of the error are given by

$$
\begin{aligned}
& \left\|\mathbf{q}-\mathbf{q}^{h}\right\|_{L^{2}}=\left\{\sum_{i=1}^{N_{e}} A_{i}\left(\mathbf{q}_{i}-\mathbf{q}_{i}^{h}\right)^{2}\right\}^{\frac{1}{2}}, \\
& \left\|\phi-\phi^{h}\right\|_{L^{2}}=\left\{\sum_{i=1}^{N_{e}} A_{i}\left(\phi_{i}-\phi_{i}^{h}\right)^{2}\right\}^{\frac{1}{2}},
\end{aligned}
$$

where $A_{i}$ is the area of the $i$ th finite element and $\mathbf{q}$ is evaluated at the centroid of each finite element using Darcy's Law. The numerically computed fluxes (normal components of the flux at the edge mid-sides) are post-processed to obtain values for $\mathbf{q}^{h}=\left(q_{x}, q_{y}\right)^{h}$ at each element centroid. The analytical and numerical potential solution is evaluated at the centroid of each finite element.

The same experiments presented in this section are reported for structured and distorted rectangular meshes in $\S 6.7$.

\subsection{Problem 1: heterogeneous, isotropic and diagonal $\mathcal{C}$}

The first test problem is similar to the one presented in Kaasschieter and Huijben [5]. The conductivity coefficient is isotropic but heterogeneous (i.e. it varies spatially) and it is given by

$$
\mathbf{K}=\left[\begin{array}{cc}
a(\mathbf{x}) & 0 \\
0 & a(\mathbf{x})
\end{array}\right]
$$

where

$$
a(\mathbf{x})=\frac{1}{1+2 \epsilon \cos (\pi x) \cos (\pi y)+\epsilon^{2} \cos ^{2}(\pi y)} .
$$

Given a source term $f=0$ and boundary conditions defined by

$$
g_{D}(\mathbf{x})=\pi(1-y), \quad \mathbf{x} \in \Gamma_{D}
$$

where $\Gamma_{D}=\{\mathbf{x} \in \Gamma: y=0$ or $y=1\}$, and

$$
g_{N}(\mathbf{x})=0, \quad \mathbf{x} \in \Gamma_{N}
$$

where $\Gamma_{N}=\{\mathbf{x} \in \Gamma: x=0$ or $x=1\}$, the boundary value problem (1) has potential and velocity solutions given by

$$
\begin{aligned}
& u(\mathbf{x})=\pi(1-y)-\epsilon \cos (\pi x) \sin (\pi y) \\
& \mathbf{q}(\mathbf{x})=-a(\mathbf{x})\left(\begin{array}{c}
\pi \epsilon \sin (\pi x) \sin (\pi y) \\
-\pi-\epsilon \cos (\pi x) \cos (\pi y)
\end{array}\right) .
\end{aligned}
$$


The potential and velocity approximations generated using the MFEM with $h=\frac{1}{32}$ and $\epsilon=0.9$ are depicted in Figure 1 .

Table 1 presents the $L^{2}$ norm of the error for the potential and velocity components. The error behaviour is in agreement with results presented by other authors [5] and with theoretical results [8]. Second order convergence, $\mathcal{O}\left(h^{2}\right)$, is observed for the potential and first order convergence, $\mathcal{O}(h)$, for velocity.

The conductivity coefficient varies from $(1-\epsilon)^{-2}$ to $(1+\epsilon)^{-2}$. When $\epsilon \rightarrow 1$, $a(\mathbf{x})$ becomes singular and therefore the rate of convergence of the potential and velocity approximations deteriorates significantly (see Table 2 and 3). In fact, for $\epsilon=0.999$ the $y$ component of the velocity approximation does not converge. An analysis of the error distribution for the velocity components reveals that it is concentrated in the upper left and lower right corners of the domain. This location corresponds to the regions where the highest variation in the coefficient $a(\mathbf{x})$ occurs (see Figure 1b). This limitation can be resolved with local mesh refinement in the upper-left and lower-right regions of the domain.

The numerical experiments using Krylov subspace methods for problem 1 are reported in Table 4 . The table includes the number of iterations required to attain convergence, $N_{i t}$, and the solution timings. For CG, the set-up time for the preconditioner, i.e. the time required to perform the incomplete Cholesky factorisation of the coefficient matrix, is reported separately ("set-up time" + "solution time").

The post-processing time (MHFEM only) whereby the potential and velocity solutions are obtained from the Lagrange multipliers solution should also be considered. However, this is negligible compared with the set-up and solution times reported in Table 4 . In fact, for the case of a fine mesh $(h=1 / 256)$ the post-processing time was calculated to be only 0.15 seconds.

The data reported in Table 4 can be summarised as follow:

1. MINRES, equipped with the Schur complement preconditioner (31) is $h$ optimal and $\mathcal{C}$-optimal, when $\mathcal{C}$ is an isotropic diagonal tensor;

2. CG using an incomplete Cholesky factorization of the coefficient matrix $D$ as preconditioner, is $\mathcal{C}$-optimal but not $h$-optimal since $N_{i t}$ grows linearly with $h$ leading to large CPU times on fine meshes;

3. On average the CPU cost per PCG iteration is lower than that required for preconditioned MINRES. Although this is a significant advantage of PCG, it is the overall number of iterations $N_{i t}$ which determines the total CPU cost $\left(t_{C P U}\right)$;

4. The results presented indicate that heterogeneity has no effect on the performance of preconditioned MINRES. Conversely, although relatively small, an increase in $N_{i t}$ and consequently $t_{C P U}$ is recorded using PCG for both small and large values of $\epsilon$.

The results of numerical experiments using algebraic multigrid as a preconditioner are presented in Table 5. The AMG set-up time, i.e. the time required to construct the coarse grids for the approximation is reported separately ("set-up time" + "solution time"). The main results can be summarised as follows: 
1. Both AMG versions of the solvers are $h$-optimal and $\mathcal{C}$-optimal;

2. As for experiments presented in Table 4, heterogeneity has no negative effect on the performance of either of the solvers;

3. Inverting the Schur complement using one V-cycle of AMG code is more efficient than using sparse direct solvers. Thus lower CPU times than those recorded in Table 4 are obtained even though the number of MINRES iterations is larger;

4. Compared to Table 4, CG solution times and iteration counts are significantly reduced when one V-cycle of AMG is used to approximately invert the MHFEM coefficient matrix;

5. The computational efficiency of the AMG versions of the solvers is partly nullified by the large CPU time required to construct the coarse grids. This CPU cost grows linearly with the mesh size;

6. The AMG coarsening process implemented on the MHFEM linear system is twice as expensive as the one implemented on the Schur complement system.

For Problem 1 on triangular meshes, MFEM with MINRES is more efficient than MHFEM.

\subsection{Problem 2: heterogeneous, anisotropic and diagonal $\mathcal{C}$}

The second test problem considers an heterogeneous, anisotropic and diagonal tensor. The conductivity coefficient $\mathcal{C}(\mathbf{x})$ is given by

$$
\mathcal{C}(\mathbf{x})=\left[\begin{array}{cc}
\alpha x^{2}+y^{2} & 0 \\
0 & x^{2}+y^{2}
\end{array}\right]
$$

The anisotropy degree of the conductivity field varies depending on the value of the coefficient $\alpha$. When $\alpha=1$, the conductivity field is isotropic. The potential and velocity solutions are chosen so that homogeneous Dirichlet boundary conditions are prescribed on $\Gamma$. These solutions are

$$
\begin{gathered}
u(\mathbf{x})=\left(x-x^{2}\right)\left(y-y^{2}\right), \\
\mathbf{q}(\mathbf{x})=-\left(\begin{array}{c}
\left(y^{2}+x^{2} \alpha\right)(-1+2 x) y(-1+y) \\
\left(x^{2}+y^{2}\right) x(-1+x)(-1+2 y)
\end{array}\right) .
\end{gathered}
$$

The source term is obtained by substituting (53) and (52) in (1) which gives

$$
\begin{aligned}
f(\mathbf{x})=- & 2 x \alpha y+2 x \alpha y^{2}+6 x^{2} \alpha y-6 x^{2} \alpha y^{2} \\
& +2 y^{3}-2 y^{4}-2 x y+6 x y^{2}+2 x^{2} y-6 x^{2} y^{2}+2 x^{3}-2 x^{4} .
\end{aligned}
$$

The MFEM potential and velocity approximations for $\alpha=1$ are depicted in Figure 2(a). The source term corresponding to (54) is illustrated in Figure 2(b).

Tables 6,7 and 8 present the $L^{2}$-norms of the error for $u$ and $\mathbf{q}$ for $\alpha=$ $10^{-2}, 1,10^{2}$, respectively. As for the previous test case $\mathcal{O}\left(h^{2}\right)$ convergence is 
recorded for the potential and $\mathcal{O}(h)$ convergence for velocity. Note that, although the convergence rates are independent of $\alpha$, the absolute errors are two orders of magnitude larger for the potential approximation and one order of magnitude larger for the velocity approximation when compared to the isotropic case.

The tables also include the minimum value for the potential solution, $u_{\min }$. According to (53), $u$ is always positive and ranges from 0 , at the domain boundaries, to 0.0625 at the centre of the domain. Interestingly, for large anisotropic factors $\left(\alpha=10^{2}\right)$ unphysical negative oscillations in the potential approximation are recorded (see Table 8) for all values of $h$. The same behaviour is not recorded for small values of $\alpha$ (see Table 6).

The computational cost of solving the MFEM and MHFEM linear systems for diagonal anisotropic conductivity coefficients is reported in Tables 9 and 10 . Following the same logic used for test problem 1, Table 9 reports the computational cost of MINRES using the exact version of preconditioner (31). For the MHFEM system, CG is used in conjunction with an incomplete Choleski factorisation of the coefficient matrix.

The numerical experiments were carried out with anisotropic coefficient $\alpha$ ranging from $10^{-2}$ to $10^{2}$. The main results reported in Table 9 are summarised as follows:

1. Anisotropy deteriorates the performance of both preconditioned MINRES and CG. The number of $p$-MINRES iterations for $\alpha=10^{-2}$ and $\alpha=10^{2}$ is between five to six times larger than for the isotropic case;

2. For finer meshes $\left(h=\frac{1}{256}\right)$ the factorisation of the coefficient matrix becomes increasingly costly, resulting in longer CPU times than for preconditioned MINRES;

3. In general, the solvers are not $\mathcal{C}$-optimal. However, for a fixed $\alpha$, MINRES is $h$-optimal. Conversely, CG iteration count varies largely even for a fixed $\alpha$.

The results of the numerical experiments using the AMG version of the solvers are reported in Table 10. These can be summarised as follows:

1. In contrast to the isotropic case, the overall CPU cost (AMG coarsening and MINRES solution time) is lower than the exact version (see Table 9);

2. As for test problem 1, the solution timings and iteration counts recorded for CG preconditioned by the AMG approximation of the coefficient matrix are by far the smallest among all methods considered. The AMG efficiency is partly nullified by the large cost of constructing the grids for the approximation. This is twice as much as implementing the coarsening on the Schur complement system;

3. The experiments show that, for $\alpha \neq 1$, the number of CG iterations varies slightly with respect to the isotropic case. Conversely, the MINRES iteration count is between five to six times larger.

When the conductivity coefficient is a diagonal anisotropic tensor, MINRES preconditioned by $(31)$ is not $\mathcal{C}$-optimal. The reason for this can be explained 
with reference to the structure of the element stiffness matrix. It can be shown that, for triangular elements, each row of the element stiffness matrix is scaled with respect to both coefficients of the diagonal tensor, $\mathcal{C}$ (see Powell [44]). This causes a significant deterioration in the performance of MINRES and loss of $\mathcal{C}$-optimality compared with the situation for isotropic coefficients. Later we show that such a deterioration in performance does not occur when rectangular elements are used.

For Problem 2 on triangular meshes, MFEM with the AMG version of MINRES is more efficient than MHFEM on medium to fine meshes.

\subsection{Problem 3: heterogeneous, anisotropic and full-tensor $\mathcal{C}$}

This test problem is reported in Younes and Fontaine [38, 39], Younes et al. [70]. The conductivity field is described by a full-tensor given by

$$
\mathcal{C}(\mathbf{x})=\left[\begin{array}{ll}
y^{2}+\alpha x^{2} & (\alpha-1) x y \\
(\alpha-1) x y & x^{2}+\alpha y^{2}
\end{array}\right]
$$

The analytical solution for the potential is given by

$$
u(\mathbf{x})=\exp \left(-20 \pi\left(\left(x-\frac{1}{2}\right)^{2}+\left(y-\frac{1}{2}\right)^{2}\right),\right.
$$

and the velocity vector is obtained using Darcy's Law $q=\mathcal{C} \nabla u$. The source term is obtained from $f=-\nabla \cdot \mathcal{C} \nabla u$. The MFEM potential and velocity approximations for $h=\frac{1}{32}$ are depicted in Figure 3(a) and the source term for $\alpha=1$ is illustrated in Figure $3(\mathrm{~b})$. Note that the source term is symmetric about $y=x$ and that the symmetry of the numerical approximation improves with mesh refinement.

$L^{2}$ norms of the error for $\alpha=1,10^{2}, 10^{3}$ are reported in Tables 11, 12 and 13 , respectively. Second order convergence for the potential and first order convergence for the velocity is confirmed. However, the magnitude of the approximation errors increases significantly as the order of the anisotropy factor $\alpha$ increases. For $\alpha=1000$ the error in the potential and velocity approximations is three orders of magnitude larger than for the isotropic case. Hence, for large anisotropy the approximation is unphysical and should be ignored. The minimum and maximum values of the potential approximation provide evidence of this behaviour. The potential is always positive and ranges from approximately zero close to the boundaries to one at the centre of the domain. For $\alpha=10^{2}$ and $\alpha=10^{3}$ the minimum and maximum values of the numerical solution lie considerably outside the range of the analytical solution. These unphysical oscillations become less severe on finer meshes, indicating that local mesh refinement could potentially resolve this problem.

Note that spurious oscillations are also present in the isotropic case. This is in contrast to results obtained for the isotropic test case (problem 2 - see Table 7). Although this is somewhat surprising it is largely in agreement with results presented by other researchers. Younes and Fontaine [38] reported that for the same test problem spurious negative oscillations are present for isotropic and anisotropic numerical experiments not only for the MFEM but also for the 
MPFA method. However, the latest MPFA schemes on cell-centred triangles with full pressure support [71] have been tested for a range of variations using the full-tensor given by (55) and such spurious oscillations are not observed. In the isotropic case the spurious oscillations disappear with mesh refinement. In fact, when $h=1 / 512$ (not shown in Table 11) negative oscillations are of the order of $10^{-7}$. Reasons for negative oscillations in the isotropic case are not reported by Younes and Fontaine [38] and this matter requires further investigation in the future.

The computational cost of solving the linear systems of equations using $p$ MINRES and PCG is reported in Table 14. The main results of this table can be summarised as follows:

1. As previously observed for test problem 2 , for large degrees of anisotropy the performance of the MINRES solver deteriorates significantly;

2. Conversely, CG behaves quite differently for full tensor coefficients. Namely, CG solution timings and iteration counts seems to decrease with increasing $\alpha$. This behaviour is considered to be problem related;

3. For small and medium size meshes, PCG is largely more efficient than $p$-MINRES for large values of $\alpha$. However, on finer meshes $(h=1 / 256)$ the cost of implementing the Choleski factorisation grows significantly and the relative performance of the two methods depends on the value of $\alpha$.

The numerical experiments results using AMG are reported in Table 15. These can be summarised as follows:

1. The efficiency of the iterative solvers when used with AMG preconditioners is confirmed also for problems with general full tensor coefficients;

2. In contrast to Table 14, the number of CG iterations and solution timings increases with increasing anisotropic coefficient;

3. The CG iteration count is between seven to twenty-one times larger than the reference isotropic case, $\alpha=1$. This differs significantly from the results recorded for diagonal anisotropic coefficients and indicates that the AMG approximation of the coefficient matrix is not a robust preconditioner for CG when general full-tensor coefficients are used;

4. As for Table 14 it is evident that no one solver consistently outperforms the other. Instead, the solvers' performance depends on the size of the mesh and the degree of anisotropy. Thus $p$-MINRES ${ }_{A M G}$ performs better on fine meshes $(h=1 / 256)$, while $\mathrm{PCG}_{A M G}$ performs better on medium to coarse meshes.

For Problem 3 on triangular meshes, the relative efficiency of MFEM and MINRES is dependent on mesh size.

\subsection{Problem 4: discontinuous, anisotropic and full-tensor $\mathcal{C}$}

This test problem was originally presented in Crumpton et al. [72]. This example will be used to assess the efficiency and accuracy of MFEM for cases 
in which the conductivity coefficient is strongly discontinuous. This is a situation which is very often encountered in applications and therefore of significant importance in this field.

Define $D=[-1,1]^{2}$ and the conductivity coefficient $\mathcal{C}$ by

$$
\mathcal{C}=\left[\begin{array}{ll}
1 & 0 \\
0 & 1
\end{array}\right] \text { for } x<0, \quad \mathcal{C}=\alpha\left[\begin{array}{ll}
2 & 1 \\
1 & 2
\end{array}\right] \text { for } x>0 .
$$

The parameter $\alpha$ controls the strength of the discontinuity at $x=0$. The exact solution for this test problem is given by

$$
u(\mathbf{x})= \begin{cases}(2 \sin (y)+\cos (y)) \alpha x+\sin (y) & \text { for } x<0, \\ \exp (x) \sin (y) & \text { for } x>0 .\end{cases}
$$

The MFEM approximations for $\alpha=1$ and $\alpha=100$, for $h=1 / 32$ are shown in Figure 4.

$L^{2}$-norms of the error for $\alpha=1$ are presented in Table 16. For this test problem we observe the loss of one order of magnitude in the rate of convergence for the potential. However, the velocity approximation retains the characteristic first order convergence rate which was also reported for the other test problems. The maximum error in the potential approximation is located at the discontinuity and it vanishes as $h$ is progressively refined. Local mesh refinement at the location of the discontinuity should enhance the rate of convergence for the potential.

Tables 17 and 18 report $L^{2}$-norms of the error for $\alpha=10^{1}$ and $\alpha=10^{2}$. Interestingly, the magnitude of the errors in the potential are of the same order as those reported for $\alpha=1$. In contrast, the velocity errors are one and two orders larger, respectively. Noticeably, the potential convergence rate is slightly lower than one for $\alpha=10$ and approaches $\mathcal{O}\left(h^{\frac{3}{2}}\right)$ for $\alpha=100$.

The performance of the solver for test problem 4 is reported in Tables 19 and 20. The results reported in these two tables can be summarised as follows:

1. MINRES iteration count for problems with discontinuities is larger (between $30 \%$ to $40 \%$ ) than for continuous problems. The same behaviour is observed for the exact and approximated versions of preconditioner (31);

2. It appears that the exact version of $p$-MINRES is by far the most efficient solver for problems with discontinuities i.e. the solution of the problem based onMFEM using MINRES is more efficient than that based on MHFEM using CG. For all other solvers considered the CPU time required either to implement the factorisation or to construct the coarse grids has a detrimental effect on the overall performance of the solvers;

3 . For all methods the magnitude (governed by $\alpha$ ) of the discontinuity has virtually no effect on the performance of the solvers. It appears that for larger $\alpha$, i.e. sharper variation in the conductivity at the discontinuity, the number of iterations is smaller than for smaller $\alpha$, i.e. more homogeneous conditions at the discontinuity.

For Problem 4 on triangular meshes, MFEM with MINRES is clearly the most efficient method. 


\subsection{Problem 5: distorted triangular mesh}

In this section the behaviour of the numerical methods on distorted meshes is assessed. Although the mesh is distorted the finite element connectivity is regular, i.e. any node has the same number of neighbouring nodes. The test problem is taken from Arnold et al. [73]. The analytical solution on the unit square domain is $u=x(1-x) y(1-y)$. The conductivity coefficient is a unit scalar. Therefore, in this case (1) simplifies to Poisson's equation.

The distorted mesh is created by perturbing the node coordinates of the original structured mesh according to

$$
\mathbf{x}_{\text {unst }}=\mathbf{x}_{s t}+\mathbf{z} h^{\alpha}
$$

where $\mathbf{z}$ is a vector of uniformly distributed random real numbers sampled in the interval $[-0.5,0.5]$ and $\alpha$ regulates the order of the perturbation. Distorted meshes are created at each discretisation level. Examples of structured and distorted meshes used for this test problem are shown in Figure 5. For the experiments herein considered $\alpha=1.2$.

$L^{2}$-norms of the error for the structured and distorted meshes are reported in Table 21. It appears that the magnitude of the errors and the convergence rate are not affected by the irregular meshing. Hence the potential converges with rate $\mathcal{O}\left(h^{2}\right)$ and velocity with rate $\mathcal{O}(h)$. It is clear that the mixed method can also provide accurate approximations on distorted meshes.

The performance of the solvers is reported in Tables 22 and 23. The results reported in the tables can be summarised as follows:

1. Both versions of preconditioned MINRES are $h$-optimal. For the distorted case the iteration count is slightly larger and some small variations with $h$ are recorded;

2. CG using the incomplete Choleski factorisation of the coefficient matrix is not $h$-optimal. Also for this solver a larger iteration count is recorded for distorted meshes;

3. The AMG version of $\mathrm{CG}$ is $h$-optimal. Similarly to the test problems previously considered, the performance of $\mathrm{CG}$ is penalised by the large CPU cost of creating the AMG grids.

For Problem 5 on triangular meshes, MFEM with MINRES is clearly the most efficient method.

\subsection{Rectangular Meshes}

In this section the numerical experiments performed in the previous section on triangular meshes are repeated on rectangular meshes. As we will see there are some major differences with respect to the triangular case.

\subsection{Problem 1: heterogeneous, isotropic and diagonal $\mathcal{C}$}

Tables 24, 25 and 26 provide $L^{2}$-norms of the error for $\epsilon=0.9,0.99,0.999$, respectively. For the case of small heterogeneity, i.e. $\epsilon=0.9$, the approximations converge at a rate greater than two. In fact, the convergence rate for the 
potential is $\mathcal{O}\left(h^{2.08}\right)$ and for the $x$ and $y$ components of the velocity it is $\mathcal{O}\left(h^{2.16}\right)$ and $\mathcal{O}\left(h^{2.21}\right)$, respectively. This is significantly different from the convergence rates observed on triangular meshes, on which only first order convergence was recorded for the velocity approximation.

Furthermore, for the same value of $h$, the magnitude of the error in the potential for the rectangular case is lower than the triangular case. For the velocity approximation it is two orders of magnitude lower.

For the case of moderate heterogeneity, i.e. $\epsilon=0.99$, larger convergence rates are recorded for the potential, $\mathcal{O}\left(h^{2.28}\right)$. However the velocity components converge at rates $\mathcal{O}\left(h^{1.02}\right)$ and $\mathcal{O}\left(h^{1.46}\right)$, respectively. Although these rates are lower than for the case of $\epsilon=0.9$, they are still a significant improvement on those obtained for the triangular case.

For the case $\epsilon=0.999$ the convergence rates and the magnitude of the error are comparable to those recorded for the triangular case.

The performance of the solvers is recorded in Table 27. The same findings summarised in $\S 6.2$ for triangular meshes also apply to rectangular meshes. In addition to those it should be noted that:

1. The CPU timings for the solvers for the rectangular case are significantly lower than the triangular case. This is obviously associated with the smaller size of the coefficient matrix in the former case. For the same reason the cost of implementing the Choleski factorisation is considerably cheaper;

2. The iteration count for $p$-MINRES in the rectangular case is comparable to the triangular case. Although a slightly larger variability is recorded, $h$-optimality and $\mathcal{C}$-optimality is preserved;

3. In contrast to the MINRES solver, the CG iteration count for the rectangular case is significantly lower than the triangular case.

The results for the AMG experiments are reported in Table 28. The considerations highlighted in $§ 3.2 .1$ regarding Table 5 are equally valid for rectangular meshes. Additionally we note that:

1. The CPU cost of constructing the AMG grids is significantly cheaper than the triangular case. Specifically, it is cheaper by a factor of four for the Schur complement and three for the MHFEM coefficient matrix;

2. Given the smaller size of the system of equations, MINRES and CG CPU times are significantly lower than the triangular case;

3. For isotropic coefficients, the AMG versions of MINRES and CG are efficient and robust. However, their overall performance is penalised by the $\mathrm{CPU}$ cost of creating the AMG grids which is not negligible even on rectangular meshes.

For Problem 1 on rectangular meshes, MFEM with MINRES is more efficient than MHFEM. 


\subsection{Problem 2: heterogeneous, anisotropic and diagonal $\mathcal{C}$}

The settings for this test problem are described in $\$ 6.3$. The error estimates on rectangular meshes are reported in Tables 29,30 and 31 for $\alpha=10^{-2}, 1,10^{2}$. The rate of convergence is found to be $\mathcal{O}\left(h^{2}\right)$ for the potential and velocity approximations. Note that the same convergence rates are obtained independent of the value of the anisotropic coefficient, $\alpha$. Furthermore the errors are approximately of the same order of magnitude.

As explained for the triangular case, the potential solution for this test problem is always positive and specifically it is 0 at the boundaries and 0.0625 at the centre of the domain, so that $0<u<0.0625$ in $D$. On triangular meshes and for $\alpha=100$ (see Table 8), the numerical solution displays unphysical negative oscillations. According to results shown in Tables 29, 30 and 31, the potential approximation does not exhibit this erroneous behaviour on rectangular meshes.

The computational performance of the solvers on problem 2 on rectangular meshes is presented in Table 32 and can be summarised as follows:

1. As opposed to the experiments carried out on triangular meshes (see Table 9 ), preconditioned MINRES is $\mathcal{C}$-optimal when the conductivity coefficient is diagonal and anisotropic;

2. The performance of MINRES (in terms of $N_{i t}$ and $t_{C P U}$ ) is completely independent to the degree of anisotropy of the conductivity coefficient;

3. The performance of $C G$ is comparable to that reported for triangular meshes, i.e. it is neither $h$ nor $\mathcal{C}$ optimal.

The results for the AMG numerical experiments are reported in Table 33. The optimality of preconditioned MINRES, previously discussed, is also valid when the Schur complement is approximated by one V-cycle of black-box AMG. In addition to this, it is evident from Table 33 that:

1. In contrast to the experiments on triangular meshes, the number of MINRES iterations is approximately constant for $\alpha \neq 1$. Not surprisingly, for the isotropic case $(\alpha=1), N_{i t}$ is generally lower;

2. Conversely to the experiments on triangular meshes (see Table 10), for $\alpha \neq 1$ the number of CG iterations varies considerably. Reasons for the difference in performance between triangular and rectangular meshes are given below.

As pointed out by results reported in Table 33, the number of CG iterations varies considerably for $\alpha \neq 1$. This is due to the fact that the coefficient matrix $D$ is not an $M$-matrix for anisotropic diagonal tensors and rectangular meshes [44]. The black-box AMG code used in this work (and other available in the public domain Boyle et al. $[68,69])$ is set up to work with $M$-matrices. When this condition is violated the performance of black-box AMG can deteriorate significantly.

For triangular elements with diagonal-anisotropic coefficients, the Lagrange multiplier system $D$ is always an $M$-matrix, hence the behaviour of AMG is not erratic and the number of CG iterations tends to vary only slightly for $\alpha \neq 1$ 
(see Table 10). Furthermore, as proved by Powell [44], the Schur complement $\left(B A B^{T}\right)$, which is used as preconditioner for MINRES, is always an $M$-matrix, hence the optimal performance of AMG is guaranteed.

Preconditioned MINRES is $\mathcal{C}$-optimal for diagonal anisotropic conductivity coefficients on rectangular meshes due to the structure of the element stiffness matrix $A_{i, j}^{K}$ 24. Powell [44], Powell and Silvester [45] showed, in fact, that for rectangular elements $A_{K}$ is a block diagonal matrix and each block is scaled by a different entry of $\mathcal{C}$. This is very different from the triangular case where every row of the element stiffness matrix $A_{i, j}^{K}$ is scaled by all entries of $\mathcal{C}$. For Problem 3 on rectangular meshes, MFEM with MINRES is more efficient than MHFEM.

\subsection{Problem 3: heterogeneous, anisotropic and full-tensor $\mathcal{C}$}

$L^{2}$-norms of the error for test problem 3 on rectangular meshes are reported in Tables 34, 35 and 36 for various values of $\alpha$. Second order convergence rates for the potential and velocity approximations are also confirmed for problems with full-tensor, anisotropic coefficients. As for triangular meshes, the magnitude of the discrete errors increases with larger anisotropic coefficients.

As for the triangular case negative oscillations in the potential approximation are also recorded for rectangular elements. Younes and Fontaine [39] reported numerical experiments using the MFEM and MPFA for the same test problem reported in this section. The authors show numerical results which are largely consistent with the results reported in Tables 34, 35 and 36, i.e. spurious negative oscillations are present not only for the anisotropic case but also for the isotropic case. For the isotropic case the spurious oscillations disappear with mesh refinement. In fact, when $h=1 / 512$ (not shown in Table 34) negative oscillations are of the order of $10^{-8}$. Reasons for negative oscillations in the isotropic case are not reported by Younes and Fontaine [39] and this matter requires further future investigation.

The performance of the solvers for test problem 3 on rectangular meshes is reported in Table 37. The main findings of this table can be summarised as follows:

1. As for triangular elements, the performance of MINRES deteriorates significantly for large values of $\alpha$;

2. Conversely, CG behaves quite differently for full tensor coefficients since the CPU cost seems to decrease with increasing $\alpha$. Similar results were obtained for triangular meshes;

3. For $\alpha \neq 1$ CG outperforms MINRES independent of the value of $h$.

Note that for $\alpha=1$, the conductivity coefficient is equivalent to that of test problem 2. The only difference between the two problems is associated with the right-hand side of the PDE. In such circumstances it is normally expected for MINRES to converge with approximately the same number of iterations. However, comparing Tables 37 and 32 for $\alpha=1$, it is evident that the number of iterations required to solve problem 3 on rectangular meshes is significantly 
lower than problem 2. This behaviour is not observed for triangular meshes (see Tables 14 and 9).

Results for the AMG numerical experiments are reported in Table 38. The most important observations from this table can be summarised as follows:

1. The MINRES iteration count grows rapidly with increasing $\alpha$, hence the solution timings are quite large. However, given that the CPU cost of constructing the coarse grids for the AMG approximation is quite cheap for rectangular meshes, $p$-MINRES MMG $_{A}$ is the solver that performs best among those considered;

2. The performance of CG on rectangular elements differs significantly from that for triangular elements. On triangular meshes, although $\mathcal{C}$-optimality is not obtained, CG is $h$-optimal for a fixed $\alpha$. On rectangular meshes neither $\mathcal{C}$ nor $h$ optimality is established. This is associated with the violation of the $M$-matrix condition for problems with full-tensor coefficients.

For Problem 3 on rectangular meshes, MFEM with the AMG version of MINRES is more efficient than MHFEM.

\subsection{Problem 4: discontinuous, anisotropic and full-tensor $\mathcal{C}$}

Table 39 reports error estimates for $\alpha=1$ for test problem 4 on rectangular meshes. The discontinuous conductivity coefficient causes the loss of one order of magnitude in the rate of convergence for both the potential and velocity approximations. Interestingly, whilst the magnitude of the errors in the potential approximation are comparable to those recorded on triangular meshes, the velocity errors tend to be one order of magnitude lower. $L^{2}$-norms of the error for $\alpha=10$ and $\alpha=100$, are listed in Tables 40 and 41. Although first order convergence rates are also recorded, the discrete errors tend to be larger with increasing $\alpha$.

The performance of the solvers for test problem 4 on rectangular meshes are reported in Table 42. The results of the experiments for the AMG version of these solvers are given in Table 43. The main findings of these two tables can be summarised as follows:

1. As for triangular meshes, the MINRES iteration count is larger for discontinuous problems than for continuous problems (see, for example, a comparison with test problem 1). The same behaviour is observed for $\mathrm{PCG}_{A M G}$ but not for PCG;

2. The degree of the discontinuity does not affect the performance of the solvers;

3. Hence the exact version of MINRES is the most efficient solver for this class of problems. However, it should be noted that the approximated version of MINRES is also very efficient given that, for rectangular meshes, the AMG set-up time is relatively small.

For very fine meshes (problems with of the order of $10^{6}-10^{7}$ degrees of freedom) the CPU cost of inverting the Schur complement exactly becomes prohibitively expensive. Hence, approximately inverting the Schur complement 
using AMG should become more efficient for those type of problems. This consideration applies to all test problems and not only to the discontinuous case.

For Problem 4 on rectangular meshes, MFEM with MINRES is more efficient than MHFEM.

\subsection{Problem 5: distorted rectangular mesh}

Distortion of rectangular meshes is obtained in a similar fashion to that explained for triangular meshes (see $\S 6.6$ and displayed in Figure 5). Although the mesh is distorted the finite element connectivity is regular, i.e. any node has the same number of neighboring nodes. $L^{2}$-norms of the error for test problem 5 on structured and distorted rectangular meshes are listed in Table 44.

On structured rectangular meshes the potential and velocity approximations converge with rate $\mathcal{O}\left(h^{2}\right)$. This confirms the results of the previous experiments (excluding discontinuous problems where velocities converge with rate $\mathcal{O}(h)$ ).

On distorted rectangular meshes the potential approximation retains second order convergence. In contrast, the experiments show the loss of one order in the convergence rates of the velocity approximation. Thus the $x$-component of the velocity converges with rate $\mathcal{O}\left(h^{1.16}\right)$ and the $y$-component with rate $\mathcal{O}\left(h^{1.31}\right)$. The loss of accuracy in the velocity approximations obtained by MFEM and MHFEM on quadrilateral meshes is well-known and solutions to this issue have been proposed by Shen [74], Arnold et al. [73] and more recently by Younes et al. [70], for example. The problem lies in the fact that the Piola transformation of vector basis functions defined on a square reference element to the actual element is not affine for quadrilateral elements [73]. This causes loss of convergence for the approximation of the fluxes. This situation does not occur on triangular elements.

The loss of convergence reported in Table 44 refers to a simple problem with unit conductivity coefficient and trivial geometry. Therefore it is expected that this deterioration would be exacerbated on problems with general coefficients and complex geometries.

The performance of the solvers for test problem 5 on structured and distorted meshes are reported in Tables 45 and 46. The findings of those tables are summarised as follows:

1. The MINRES iteration count for problems on distorted meshes is approximately twice as that for problems with structured meshes when the Schur complement is inverted exactly. For the AMG case, instead, the difference in iteration count is less marked;

2. The PCG iteration count also varies only slightly between structured and distorted meshes. The same can be stated for CG with the AMG preconditioner;

3. Once again, MINRES with the exact version of preconditioner (31) is the best performing method.

For Problem 5 on rectangular meshes, MFEM with MINRES (exact preconditioner) is more efficient than MHFEM. 


\section{Conclusions}

This paper reports on results of numerical experiments based on mixed finite element methods and compares the accuracy of the approximations through a graded series of problems with exact solutions. This allows the codes developed for this work to be validated and for the convergence behaviour of MFEM to be investigated.

We have shown that MFEM exhibits second order convergence for the potential and first order convergence for the velocity for problems with continuous conductivity coefficient tensors on structured and distorted triangular meshes. For discontinuous problems there is a loss of one order of convergence for the potential while the rate of convergence for the velocity is unaltered. The MFEM possesses second order convergence for the potential and velocity on structured rectangular meshes. The loss of approximately one order of convergence is reported for distorted meshes. For discontinuous problems there is a loss of one order of convergence for both the potential and velocity.

Although the potential must be strictly positive for the problems considered in this paper, spurious negative values are realised for problems with diagonal anisotropic and full-tensor anisotropic coefficients on triangular meshes. This behaviour problem is also present for problems with full-tensor anisotropic coefficients on rectangular meshes. Furthermore we have observed that spurious negative oscillations are present in all cases (isotropic, anisotropic full-tensor) for test problem 3 - a finding that is in agreement with the literature [38, 39]. In the isotropic case the magnitude of the oscillations tend to zero as the mesh is progressively refined.

In addition to an investigation of the influence of the nature of the conductivity coefficient on the order of convergence of the finite element approximation on triangular and quadrilateral meshes, a detailed analysis of the relative computational cost of solving the indefinite linear system obtained with MFEM with the symmetric positive definite system obtained with MHFEM is performed. For problems with isotropic, heterogeneous coefficients, the use of MINRES in which the exact Schur complement is used as the preconditioner is the most efficient method in terms of CPU cost. This was also found to be the case for problems with anisotropic diagonal tensors but only on rectangular meshes. In these cases, MINRES is $h$-optimal and $\mathcal{C}$-optimal. Thus solving the indefinite system is the cheapest approach to solving the mixed formulation in these particular instances.

The implementation of MINRES using the AMG version of the Schur complement as preconditioner also results in a very efficient iterative method. In particular, the number of iterations required to attain the convergence criterion is reduced resulting in significant CPU savings. However, one does need to account for the cost of creating the coarse grids for the AMG approximation which is not negligible irrespective of whether one is solving the Schur complement or Lagrange multiplier systems. This component of the computational

cost is more expensive for the SPD case than for the Schur complement and also on triangular meshes than on rectangular meshes. 
The optimal performance of the AMG preconditioners depends inextricably on whether the coefficient matrix satisfies the $M$-matrix condition. The Schur complement is always an $M$-matrix. Hence MINRES using the AMG version of the Schur complement preconditioner will never fail to converge. In contrast, the Lagrange multiplier system is only an $M$-matrix for problems with scalar and diagonal coefficients and when triangular elements are used. For general coefficients and triangular elements the $M$-matrix condition does not hold. Furthermore, for rectangular meshes the $M$-matrix condition does not hold under any circumstances for the SPD system. Hence, using the AMG approximation of the coefficient matrix as preconditioner for CG on rectangular meshes does not guarantee success and, potentially, it could fail to converge.

For general full-tensor conductivity coefficients the results are more difficult to summarise. It is generally the case that AMG preconditioners perform better. On rectangular meshes the Schur complement preconditioner (AMG version) is the cheapest approach among those considered. The same applies to triangular meshes on fine discretisations while the AMG approximation of the Lagrange multiplier system is the cheapest on medium to coarse meshes. However, for the latter case the success of black-box AMG depends on the extent to which the $M$-matrix condition is violated. Thus, its performance is problem dependent.

The numerical experiments that have been presented show that the solution of the indefinite system (MFEM) is often cheaper and more reliable than the solution of the SPD system (MHFEM). This is certainly the case for isotropic and diagonal-tensor conductivity coefficients. However, none of the iterative methods have been shown to be optimal for solving problems with the fulltensor conductivity coefficients considered in this paper.

\section{Acknowledgement}

The authors would like to express their gratitude to Professor E.F. Kaasschieter for his help with the computer implementation of the MHFEM and for

providing useful MATLAB functions to develop the code used for the numerical experiments presented in this paper.

\section{References}

[1] Goode, D.J.. Particle velocity interpolation in blockcentered finite difference groundwater flow models. Water Resour Res 1990;26(5):925-40.

[2] Cordes, C., Kinzelbach, W.. Continuous groundwater velocity fields and path lines in linear, bilinear, and trilinear finite elements. Water Resour Res 1992;28(11):2903-2911.

[3] Srivastava, R., Brusseau, M.L.. Darcy velocity computations in the finite element method for multidimensional randomly heterogeneous porous media. Adv Water Resour 1995;18(4):191-201. 
[4] Dogrul, E.C., Kadir, T.N.. Flow computation and mass balance in Galerkin finite-element groundwater models. J Hydr Engrg 2006;132(11):1206-1214.

[5] Kaasschieter, E.F., Huijben, A.J.M.. Mixed-hybrid finite elements and streamline computation for the potential flow problem. Numer Meth Part D E 1992;8(3):221-266.

[6] Mosé, R., Siegel, P., Ackerer, P., Chavent, G.. Application of the mixed hybrid finite element approximation in a groundwater flow model: Luxury or necessity? Water Resour Res 1994;30(11):3001-3012.

[7] Arnold, D.N., Brezzi, F.. Mixed and non-conforming finite element methods: Implementation, postprocessing and error estimates. $\mathrm{M}^{2} \mathrm{AN}$ Math Model Numer Anal 1985;19(1):7-32.

[8] Brezzi, F., Fortin, M.. Mixed and Hybrid Finite Element Methods. Springer-Verlag; 1991.

[9] Durlofsky, L.. Accuracy of mixed and control volume finite element approximations to Darcy velocity and related quantities. Water Resour Res 1994;30(4):965-973.

[10] Kaasschieter, E.F.. Mixed finite elements for accurate particle tracking in saturated groundwater flow. Adv Water Resour 1995;18(5):277-294.

[11] Raviart, P.A., Thomas, J.M.. A mixed finite element method for second-order elliptic problems. Mathematical Aspects of the Finite Element Method. In: Lect. Notes in Math.; vol. 606. Springer-Verlag; 1977, p. $292-315$.

[12] Nedelec, J.C.. Mixed finite elements in $\mathbb{R}^{3}$. Numer Math 1980;35:315-341.

[13] Chavent, G., Jaffré, J.. Mathematical Models and Finite Elements for Reservoir Simulation. North-Holland; 1986.

[14] Roberts, J.E., Thomas, J.. Mixed and hybrid methods. In: Ciarlet, P., Lions, J., editors. Handbook of Numerical Analysis. Elsevier Science Publishers; 1991, p. 523-639.

[15] Chavent, G., Roberts, J.E.. A unified physical presentation of mixed, mixed-hybrid finite elements and standard finite difference approximations for the determination of velocities in waterflow. Adv Water Resour $1991 ; 14(6): 329-348$.

[16] Brezzi, F., Jr., J.D., Marini, L.. Two families of mixed finite elements for second order elliptic problems. Numer Math 1985;47:217-235.

[17] Bause, M., Hoffmann, J., Knabner, P.. First-order convergence of multipoint flux approximation on triangular grids and comparison with mixed finite element methods. Numer Math 2010;116:1-29. 
[18] Arbogast, T., Wheeler, M.F., Zhang, N.Y.. A nonlinear mixed finite element method for a degenerate parabolic equation arising in flow in porous media. SIAM J Numer Anal 1996;33:1669-1687.

[19] Demlow, A.. Suboptimal and optimal convergence in mixed finite element methods. SIAM J Numer Anal 2002;39:1938-1953.

[20] Radu, A., Pop, I., Knabner, P.. Order of convergence estimates for an Euler implicit, mixed finite element discretization of Richards' equation. SIAM J Numer Anal 2004;42:1452-1478.

[21] Edwards, M.G., Rogers, C.F.. Finite volume discretization with imposed flux continuity for the general tensor pressure equation. Comput Geosci 1998;2(259-290).

[22] Aavatsmark, I., Barkve, T., Be, ., Mannseth, T.. Discretization on unstructured grids for inhomogeneous, anisotropic media. Part I: Derivation of the methods. SIAM J Sci Comput 1998;19:1700-1716.

[23] Aavatsmark, I., Barkve, T., Be, ., Mannseth, T.. Discretization on unstructured grids for inhomogeneous, anisotropic media. Part II: Discussion and numerical results. SIAM J Sci Comput 1998;19:1717-1736.

[24] Aavatsmark, I., Barkve, T., T.Mannseth, . Control-volume discretization methods for 3D quadrilateral grids in inhomogeneous, anisotropic reservoirs. SPE Journal 1998;3:146-154.

[25] Edwards, M.G.. Unstructured, control-volume distributed, full-tensor finite volume schemes with flow based grids. Comput Geosci 2002;6(433-452).

[26] Aavatsmark, I.. An introduction to multipoint flux approximations for quadrilateral grids. Comput Geosci 2002;6:404-432.

[27] Edwards, M.G., Pal, M.. Positive definite q-families of continuous subcell Darcy-flux CVD (MPFA) finite-volume schemes and the mixed finite element method. Int J Numer Methods Fluids 2008;57(355-387).

[28] Edwards, M.G., Zheng, H.. A quasi-positive family of continuous Darcyflux finite volume schemes with full pressure support. J Comput Phys 2008;227(9333-9364).

[29] Friis, H.A., Edwards, M.G., Mykkeltveit, J.. Symmetric positive definite flux-continuous full-tensor finite-volume schemes on unstructured cell centered triangular grids. SIAM J Sci Comput 2008;31(1192-1220).

[30] Edwards, M.G., Zheng, H.. Double-families of quasi-positive darcy-flux approximations with highly anisotropic tensors on structured and unstructured grids. J Comput Phys 2010;229(594-625). 
[31] Edwards, M.G., Zheng, H.. Quasi M-matrix multi-family continuous darcy-flux approximations with full pressure support on structured and unstructured grids in 3-d. SIAM J Sci Comput 2011;33(455-487).

[32] Chavent, G., Younes, A., H.Ackerer, P.. On the finite volume reformulation of the mixed finite element method for elliptic and parabolic PDE on triangles. Comput Meth Appl Mech Eng 2003;192:655-682.

[33] Younes, A., Ackerer, P.H., Chavent, G.. From mixed finite elements to finite volumes for elliptic PDE in 2 and 3 dimensions. Int J Numer Meth Eng 2004;59:365-388.

[34] Brezzi, F., Fortin, M., Marini, L.D.. Piecewise constant pressures for Darcy law. In: L.P., F., editor. In Finite Elements Methods: 1970 and Beyond. CIMNE: Barcelona; 2004,.

[35] Vohralik, M.. Equivalence between lowest-order mixed finite element and multi-point finite volume methods on simplicial meshes. ESAIM: M2AN 2006;40:367-391.

[36] Klausen, C., Russell, C.. Relationships among some locally conservative discretization methods which handle discontinuous coefficients. Comput Geosci 2004;8:341-377.

[37] Wheeler, M.F., Yotov, I.. A multipoint flux mixed finite element method. SIAM J Numer Anal 2006;44:2082-2106.

[38] Younes, A., Fontaine, V.. Hybrid and multi-point formulations of the lowest-order mixed methods for Darcy's flow on triangles. Int J Numer Meth Fluids 2008;58(9):1041-1062.

[39] Younes, A., Fontaine, V.. Efficiency of mixed hybrid finite element and multipoint flux approximation methods on quadrangular grids and highly anisotropic media. Int J Numer Meth Eng 2008;76(3):314-336.

[40] Younes, A., Ackerer, P.H., Mosé, R., Chavent, G.. A new formulation of the mixed finite element method for solving elliptic and parabolic PDE with triangular elements. J Comput Phys 1999;149:148-167.

[41] Paige, C.C., Saunders, M.A.. Solution of sparse indefinite systems of linear equations. SIAM J Numer Anal 1975;12:617-629.

[42] Rusten, T., Wither, R.. A preconditioned iterative method for saddlepoint problems. SIAM J Matrix Anal A 1992;13(3):887-904.

[43] Vassilevski, P.S., Lazarov, R.D.. Preconditioning mixed finite element saddle-point elliptic problems. Numer Linear Algebr 1996;3(1):1-20.

[44] Powell, C.E.. Optimal preconditioning for mixed finite element formulation of second-order elliptic problems. Ph.D. thesis; University of Manchester; 2003. 
[45] Powell, C.E., Silvester, D.. Optimal preconditioning for Raviart-Thomas mixed formulation of second-order elliptic problems. SIAM J Matrix Anal A 2003;25(3):718-738.

[46] Powell, C.E.. Parameter-free $H(d i v)$ preconditioning for mixed finite element formulation of diffusion problems. IMA J Numer Anal 2005;25(4):783-796.

[47] Scheichl, R.. Iterative solution of saddle point problems using divergencefree finite elements with applications to groundwater flow. Ph.D. thesis; University of Bath; 2000.

[48] Kim, C.. On iteration and approximation methods for anisotropic problems. Ph.D. thesis; Texas A\&M University; 2001.

[49] Ewing, R.E., Wheeler, M.F.. Computational aspects of mixed finite element methods. In: R., S., editor. Scientific Computing. IMACS, NorthHolland; 1983,.

[50] Fortin, M., Glowinski, R.. Augmented Lagrangian Methods. NorthHolland; 1983.

[51] Rusten, T., Wither, R.. Substructure preconditioners for elliptic saddle point problems. Math Comput 1993;60(201):23-48.

[52] Rusten, T., Vassilevski, P., Wither, R.. Interior preconditioners for mixed finite element approximations of elliptic problems. Math Comput 1996;65(214):447-466.

[53] de Veubeke, B.F.. Displacement and equilibrium models in the finite element method. In: Zienkiewicz, O.C., Holister, G., editors. Stress Analysis. John Wiley and Sons; 1965,.

[54] Saad, Y.. Iterative Methods for Sparse Linear Systems. SIAM; 2003.

[55] C.Eisenstat, S.. Efficient implementation of a class of conjugate gradient methods. SIAM J Sci Stat Comp 1981;2:1-4.

[56] Briggs, W.L., Henson, V.E., McCormick, S.F.. A Multigrid Tutorial. SIAM; 2000.

[57] Hackbusch, W.. Multi-Grid Methods and Applications. Berlin: SpringerVerlag; 2003.

[58] Brenner, S.C.. An optimal-order multigrid method for P1 nonconforming finite elements. Math Comput 1989;52(185):1-15.

[59] Brenner, S.C.. A multigrid algorithm for the lowest-order RaviartThomas mixed triangular finite element method. SIAM J Numer Anal 1992;29(3):647-678. 
[60] Braess, D., Verfürth, R.. Multigrid methods for nonconforming finite element methods. SIAM J Numer Anal 1990;27(4):979-986.

[61] Chen, Z.. Equivalence between and multigrid algorithms for nonconforming and mixed methods for second-order elliptic problems. East-West J Numer Math 1996;4(1):1-33.

[62] Davis, T.A., Duff, I.S.. An unsymmetric-pattern multifrontal method for sparse LU factorization. SIAM J Matrix Anal A 1997;18(1):140-158.

[63] Davis, T.A., Duff, I.S.. A combined unifrontal / multifrontal method for unsymmetric sparse matrices. ACM T Math Software 1999;25(1):1-19.

[64] A.Davis, T.. Algorithm 832: UMFPACK, an unsymmetric-pattern multifrontal method. ACM T Math Software 2004;30(2):196-199.

[65] MATLAB, . Version 7.4.0, user's guide. Tech. Rep.; The Mathworks Inc, Prentice Hall; 1997.

[66] Fischer, B.. Polynomial Based Iteration Methods for Symmetric Linear Systems. Wiley-Teubner; 1996.

[67] Silvester, D.J., Powell, C.E.. PIFISS potential (incompressible) flow \& iterative solution software guide. Tech. Rep.; University of Manchester; 2007.

[68] Boyle, J., Mihajlovic, M.D., A.Scott, J.. HSL_MI20: an efficient AMG preconditioner. Tech. Rep. RAL-TR-2007-021; University of Manchester; 2007.

[69] Boyle, J., Mihajlovic, M.D., Scott, J.A.. HSL_MI20: an efficient AMG preconditioner for finite element problems in 3D. Int J Numer Meth Eng 2009;82(1):64-98.

[70] Younes, A., Ackerer, P.H., Ahmed, S., Bouhlila, R.. A technique for improving the accuracy of quadrangular mixed finite elements for Darcy's flow on heterogeneous domains. Comput Fluids 2010;39:189-196.

[71] Friis, H.A., Edwards, M.G.. A family of MPFA finite volume schemes with full pressure support for the general tensor pressure equation on cellcentred triangular grids. J Comput Phys 2011;230(205-231).

[72] Crumpton, P.I., Shaw, G.J., Ware, A.F.. Discretisation and multigrid solution of elliptic equations with mixed derivative terms and strongly discontinuous coefficients. J Comput Phys 1995;116:343-358.

[73] Arnold, D.N., Boffi, D., Falk, R.S.. Quadrilateral $H($ div ) finite elements. SIAM J Numer Anal 2005;42(6):2429-2451.

[74] Shen, J.. Mixed finite element methods on distorted rectangular grids. Tech. Rep.; Institute for Scientific Computation, Texas A\&M University; 1994. 
Table 1: $L^{2}$-norms of the error for test problem $1, \epsilon=0.9$

\begin{tabular}{ccccccc}
\hline$h$ & $\left\|u-u^{h}\right\|_{L^{2}}$ & Rate & $\left\|q_{x}-q_{x}^{h}\right\|_{L^{2}}$ & Rate & $\left\|q_{y}-q_{y}^{h}\right\|_{L^{2}}$ & Rate \\
\hline \hline$\frac{1}{16}$ & $4.34 E-03$ & - & $2.44 E-01$ & - & $6.27 E-01$ & - \\
$\frac{1}{32}$ & $9.25 E-04$ & 2.23 & $1.15 E-01$ & 1.09 & $2.97 E-01$ & 1.08 \\
$\frac{1}{64}$ & $2.25 E-04$ & 2.04 & $5.72 E-02$ & 1.00 & $1.41 E-01$ & 1.07 \\
$\frac{1}{128}$ & $5.61 E-05$ & 2.00 & $2.85 E-02$ & 1.01 & $7.02 E-02$ & 1.01 \\
$\frac{1}{256}$ & $1.40 E-05$ & 2.00 & $1.42 E-02$ & 1.00 & $3.51 E-02$ & 1.00 \\
\hline
\end{tabular}

Table 2: $L^{2}$-norms of the error for test problem $1, \epsilon=0.99$

\begin{tabular}{ccccccc}
\hline$h$ & $\left\|u-u^{h}\right\|_{L^{2}}$ & Rate & $\left\|q_{x}-q_{x}^{h}\right\|_{L^{2}}$ & Rate & $\left\|q_{y}-q_{y}^{h}\right\|_{L^{2}}$ & Rate \\
\hline \hline$\frac{1}{16}$ & $1.09 E-02$ & - & $7.43 E-01$ & - & $1.64 E+00$ & - \\
$\frac{1}{32}$ & $3.17 E-03$ & 1.78 & $5.52 E-01$ & 0.43 & $1.64 E+00$ & 0.00 \\
$\frac{1}{64}$ & $8.19 E-04$ & 1.95 & $3.59 E-01$ & 0.62 & $1.51 E+00$ & 0.11 \\
$\frac{1}{128}$ & $1.74 E-04$ & 2.24 & $1.87 E-01$ & 0.94 & $1.20 E+00$ & 0.34 \\
$\frac{1}{256}$ & $3.27 E-05$ & 2.41 & $8.51 E-02$ & 1.13 & $6.18 E-01$ & 0.96 \\
\hline
\end{tabular}

Table 3: $L^{2}$-norms of the error for test problem $1, \epsilon=0.999$

\begin{tabular}{ccccccc}
\hline$h$ & $\left\|u-u^{h}\right\|_{L^{2}}$ & Rate & $\left\|q_{x}-q_{x}^{h}\right\|_{L^{2}}$ & Rate & $\left\|q_{y}-q_{y}^{h}\right\|_{L^{2}}$ & Rate \\
\hline \hline$\frac{1}{16}$ & $1.22 E-02$ & - & $8.28 E-01$ & - & $1.91 E+00$ & - \\
$\frac{1}{32}$ & $3.97 E-03$ & 1.62 & $6.92 E-01$ & 0.26 & $2.19 E+00$ & $<0$ \\
$\frac{1}{64}$ & $1.29 E-03$ & 1.63 & $5.74 E-01$ & 0.27 & $2.48 E+00$ & $<0$ \\
$\frac{1}{128}$ & $4.09 E-04$ & 1.65 & $4.65 E-01$ & 0.30 & $2.69 E+00$ & $<0$ \\
$\frac{1}{256}$ & $1.25 E-04$ & 1.72 & $3.57 E-01$ & 0.38 & $2.75 E+00$ & $<0$ \\
\hline
\end{tabular}

Table 4: Iteration count and timings (set-up and solution) for $p-M I N R E S$ and PCG - Test problem 1

\begin{tabular}{ccccccc}
\hline & & \multicolumn{2}{c}{$p-M I N R E S$} & & \multicolumn{2}{c}{$P C G$} \\
\cline { 3 - 4 } \cline { 6 - 7 }$h$ & $\epsilon$ & $N_{i t}$ & $t_{C P U}$ & & $N_{i t}$ & $t_{C P U}$ \\
\hline \hline$\frac{1}{64}$ & $\epsilon=0.999$ & 43 & 0.97 & & 135 & $1.11+1.35$ \\
& $\epsilon=0.99$ & 43 & 0.97 & & 139 & $1.10+1.42$ \\
& $\epsilon=0.9$ & 44 & 1.03 & & 138 & $1.11+1.42$ \\
\hline$\frac{1}{128}$ & $\epsilon=0.999$ & 43 & 5.54 & & 256 & $17.97+11.63$ \\
& $\epsilon=0.99$ & 43 & 5.46 & & 255 & $17.99+11.87$ \\
& $\epsilon=0.9$ & 43 & 5.58 & & 270 & $18.09+12.10$ \\
\hline$\frac{1}{256}$ & $\epsilon=0.999$ & 43 & 28.38 & & 525 & $285.16+113.93$ \\
& $\epsilon=0.99$ & 43 & 28.34 & & 495 & $281.56+108.26$ \\
& $\epsilon=0.9$ & 43 & 28.36 & & 535 & $284.56+117.26$ \\
\hline
\end{tabular}


Table 5: Iteration count and timings (set-up and solution) for $p-M I N R E S_{A M G}$ and $P C G_{A M G}$ - Test problem 1

\begin{tabular}{|c|c|c|c|c|c|}
\hline \multirow[b]{2}{*}{$h$} & \multirow[b]{2}{*}{$\epsilon$} & \multicolumn{2}{|c|}{$p-M I N R E S_{A M G}$} & \multicolumn{2}{|c|}{$P C G_{A M G}$} \\
\hline & & $N_{i t}$ & $t_{C P U}$ & $N_{i t}$ & $t_{C P U}$ \\
\hline \multirow{3}{*}{$\frac{1}{64}$} & $\epsilon=0.999$ & 49 & $1.33+0.98$ & 9 & $1.93+0.13$ \\
\hline & $\epsilon=0.99$ & 51 & $1.37+1.05$ & 9 & $1.94+0.13$ \\
\hline & $\epsilon=0.9$ & 51 & $1.29+1.04$ & 9 & $1.95+0.13$ \\
\hline \multirow{3}{*}{$\frac{1}{128}$} & $\epsilon=0.999$ & 51 & $8.04+4.21$ & 10 & $13.98+0.50$ \\
\hline & $\epsilon=0.99$ & 52 & $8.28+4.38$ & 9 & $14.10+0.43$ \\
\hline & $\epsilon=0.9$ & 51 & $8.04+4.28$ & 9 & $13.88+0.42$ \\
\hline \multirow{3}{*}{$\frac{1}{256}$} & $\epsilon=0.999$ & 56 & $110.60+22.42$ & 9 & $269.54+2.20$ \\
\hline & $\epsilon=0.99$ & 56 & $109.60+22.62$ & 10 & $281.54+2.34$ \\
\hline & $\epsilon=0.9$ & 54 & $108.31+22.03$ & 9 & $268.47+2.29$ \\
\hline
\end{tabular}

Table 6: $L^{2}$-norms of the error for test problem 2, $\alpha=10^{-2}$

\begin{tabular}{cccccccc}
\hline$h$ & $\left\|u-u^{h}\right\|_{L^{2}}$ & Rate & $\left\|q_{x}-q_{x}^{h}\right\|_{L^{2}}$ & Rate & $\left\|q_{y}-q_{y}^{h}\right\|_{L^{2}}$ & Rate & $u_{\min }$ \\
\hline \hline$\frac{1}{16}$ & $1.05 E-03$ & - & $3.44 E-03$ & - & $4.14 E-03$ & - & $3.15 E-04$ \\
$\frac{1}{32}$ & $2.80 E-04$ & 1.91 & $1.73 E-03$ & 0.99 & $2.09 E-03$ & 0.99 & $8.02 E-05$ \\
$\frac{1}{64}$ & $7.11 E-05$ & 1.98 & $8.69 E-04$ & 1.00 & $1.05 E-03$ & 1.00 & $2.02 E-05$ \\
$\frac{1}{128}$ & $1.78 E-05$ & 2.00 & $4.35 E-04$ & 1.00 & $5.24 E-04$ & 1.00 & $5.07 E-06$ \\
$\frac{1}{256}$ & $4.46 E-06$ & 2.00 & $2.17 E-04$ & 1.00 & $2.62 E-04$ & 1.00 & $1.27 E-06$ \\
\hline
\end{tabular}

Table 7: $L^{2}$-norms of the error for test problem 2, $\alpha=1$

\begin{tabular}{cccccccc}
\hline$h$ & $\left\|u-u^{h}\right\|_{L^{2}}$ & Rate & $\left\|q_{x}-q_{x}^{h}\right\|_{L^{2}}$ & Rate & $\left\|q_{y}-q_{y}^{h}\right\|_{L^{2}}$ & Rate & $u_{\min }$ \\
\hline \hline$\frac{1}{16}$ & $1.85 E-04$ & - & $4.32 E-03$ & - & $4.32 E-03$ & - & $3.22 E-04$ \\
$\frac{1}{32}$ & $4.65 E-05$ & 1.99 & $2.18 E-03$ & 0.99 & $2.18 E-03$ & 0.99 & $8.11 E-05$ \\
$\frac{1}{64}$ & $1.16 E-05$ & 2.00 & $1.09 E-03$ & 1.00 & $1.09 E-03$ & 1.00 & $2.03 E-05$ \\
$\frac{1}{128}$ & $2.90 E-06$ & 2.00 & $5.47 E-04$ & 1.00 & $5.47 E-04$ & 1.00 & $5.08 E-06$ \\
$\frac{1}{256}$ & $7.26 E-07$ & 2.00 & $2.74 E-04$ & 1.00 & $2.74 E-04$ & 1.00 & $1.27 E-06$ \\
\hline
\end{tabular}

Table 8: $L^{2}$-norms of the error for test problem 2, $\alpha=10^{2}$

\begin{tabular}{cccccccc}
\hline$h$ & $\left\|u-u^{h}\right\|_{L^{2}}$ & Rate & $\left\|q_{x}-q_{x}^{h}\right\|_{L^{2}}$ & Rate & $\left\|q_{y}-q_{y}^{h}\right\|_{L^{2}}$ & Rate & $u_{\min }$ \\
\hline \hline$\frac{1}{16}$ & $5.38 E-03$ & - & $3.57 E-01$ & - & $3.00 E-01$ & - & $-8.10 E-03$ \\
$\frac{1}{32}$ & $1.35 E-03$ & 1.99 & $1.79 E-01$ & 0.99 & $1.51 E-01$ & 0.99 & $-2.19 E-03$ \\
$\frac{1}{64}$ & $3.39 E-04$ & 2.00 & $8.97 E-02$ & 1.00 & $7.53 E-02$ & 1.00 & $-5.65 E-04$ \\
$\frac{1}{128}$ & $8.47 E-05$ & 2.00 & $4.49 E-02$ & 1.00 & $3.77 E-02$ & 1.00 & $-1.43 E-04$ \\
$\frac{1}{256}$ & $2.12 E-05$ & 2.00 & $2.24 E-02$ & 1.00 & $1.88 E-02$ & 1.00 & $-3.58 E-05$ \\
\hline
\end{tabular}


Table 9: Iteration count and timings (set-up and solution time) for $p-M I N R E S$ and PCG - Test problem 2

\begin{tabular}{ccccccc}
\hline \multirow{2}{*}{$h$} & & \multicolumn{2}{c}{$p-$ MINRES } & & \multicolumn{2}{c}{$P C G$} \\
\cline { 3 - 4 } \cline { 6 - 6 } & $\alpha$ & $N_{i t}$ & $t_{C P U}$ & & $N_{i t}$ & $t_{C P U}$ \\
\hline \hline$\frac{1}{64}$ & $\alpha=10^{2}$ & 240 & 5.93 & & 78 & $0.99+0.92$ \\
& $\alpha=1$ & 43 & 0.82 & & 112 & $1.08+1.13$ \\
& $\alpha=10^{-2}$ & 211 & 5.10 & & 110 & $1.03+1.10$ \\
\hline$\frac{1}{128}$ & $\alpha=10^{2}$ & 246 & 32.51 & & 155 & $15.80+6.94$ \\
& $\alpha=1$ & 43 & 5.40 & & 219 & $17.45+10.03$ \\
& $\alpha=10^{-2}$ & 226 & 29.66 & & 225 & $16.39+10.38$ \\
\hline$\frac{1}{256}$ & $\alpha=10^{2}$ & 248 & 166.29 & & 313 & $242.93+67.91$ \\
& $\alpha=1$ & 43 & 28.68 & & 435 & $266.38+94.77$ \\
& $\alpha=10^{-2}$ & 233 & 155.29 & & 465 & $248.99+100.43$ \\
\hline
\end{tabular}

Table 10: Iteration count and timings (set-up and solution time) for $p-M I N R E S_{A M G}$ and $P C G_{A M G}$ - Test problem 2

\begin{tabular}{ccccccc}
\hline \multirow{2}{*}{$h$} & \multirow{2}{*}{$\alpha$} & \multicolumn{2}{c}{$p-M I N R E S_{A M G}$} & & \multicolumn{2}{c}{$P C G_{A M G}$} \\
\cline { 3 - 4 } \cline { 6 - 7 } & $N_{i t}$ & $t_{C P U}$ & & $N_{i t}$ & $t_{C P U}$ \\
\hline \hline$\frac{1}{64}$ & $\alpha=10^{2}$ & 235 & 2.81 & & 10 & $1.96+0.15$ \\
& $\alpha=1$ & 50 & 0.61 & & 9 & $1.96+0.13$ \\
& $\alpha=10^{-2}$ & 212 & 2.51 & & 11 & $2.04+0.15$ \\
\hline$\frac{1}{128}$ & $\alpha=10^{2}$ & 242 & 10.56 & & 12 & $12.75+0.56$ \\
& $\alpha=1$ & 52 & 2.17 & & 9 & $14.36+0.44$ \\
$\frac{1}{256}$ & $\alpha=10^{-2}$ & 227 & 10.11 & & 12 & $14.01+0.56$ \\
& $\alpha=10^{2}$ & 245 & 54.91 & & 13 & $241.33+3.18$ \\
& $\alpha=1$ & 54 & 11.73 & & 10 & $256.18+2.43$ \\
& $\alpha=10^{-2}$ & 232 & 52.73 & & 12 & $251.11+2.88$ \\
\hline
\end{tabular}



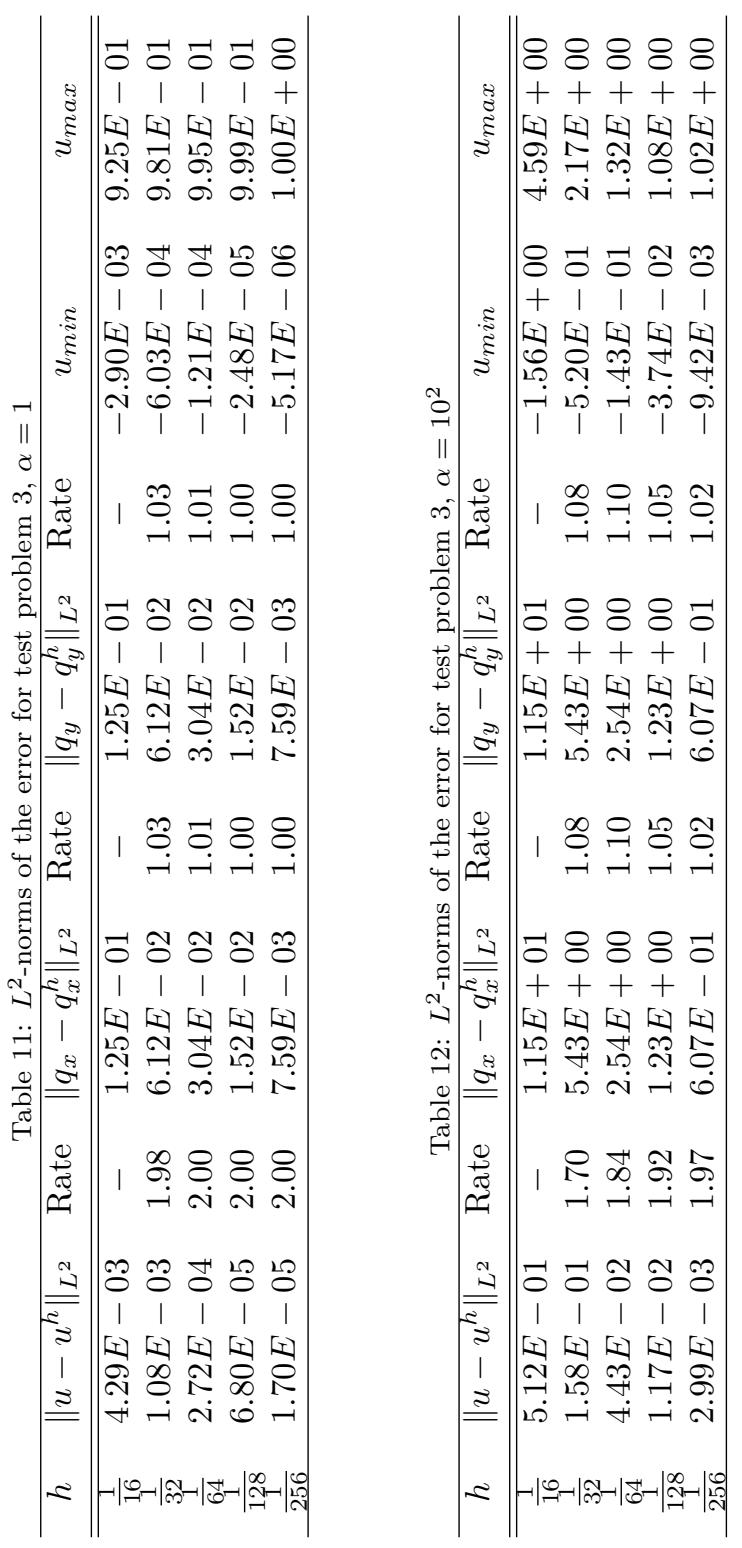


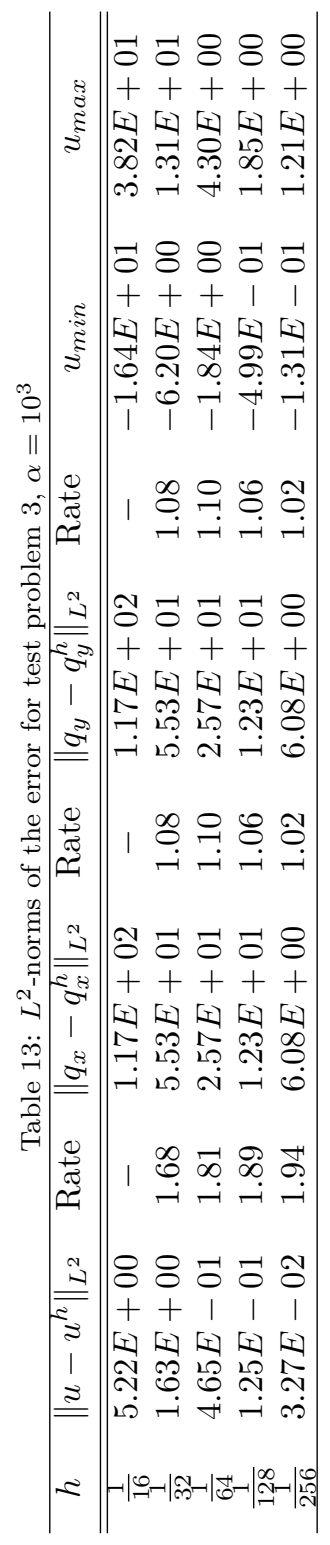


Table 14: Iteration count and timings (set-up and solution time) for $p-M I N R E S$ and PCG - Test problem 3

\begin{tabular}{ccccccc}
\hline \multirow{2}{nnnnnyy}{$h$} & & \multicolumn{2}{c}{$p-M I N R E S$} & & \multicolumn{2}{c}{$P C G$} \\
\cline { 3 - 4 } \cline { 6 - 7 } & $\alpha$ & $N_{i t}$ & $t_{C P U}$ & & $N_{i t}$ & $t_{C P U}$ \\
\hline \hline$\frac{1}{64}$ & $\alpha=10^{3}$ & 460 & 11.57 & & 12 & $0.94+0.16$ \\
& $\alpha=10^{2}$ & 271 & 6.61 & & 21 & $0.95+0.24$ \\
& $\alpha=1$ & 43 & 1.01 & & 113 & $1.10+1.17$ \\
\hline$\frac{1}{128}$ & $\alpha=10^{3}$ & 380 & 49.37 & & 14 & $15.56+0.67$ \\
& $\alpha=10^{2}$ & 316 & 41.25 & & 37 & $15.36+1.73$ \\
& $\alpha=1$ & 45 & 5.69 & & 220 & $17.09+10.02$ \\
\hline$\frac{1}{256}$ & $\alpha=10^{3}$ & 474 & 316.87 & & 18 & $238.50+4.13$ \\
& $\alpha=10^{2}$ & 334 & 222.67 & & 73 & $238.48+16.31$ \\
& $\alpha=1$ & 45 & 29.40 & & 441 & $266.61+97.68$ \\
\hline
\end{tabular}

Table 15: Iteration count and timings (set-up and solution times) for $p-M I N R E S_{A M G}$ and $P C G_{A M G}$ - Test problem 3

\begin{tabular}{ccccccc}
\hline \multirow{2}{*}{$h$} & & \multicolumn{2}{c}{$p-M I N R E S_{A M G}$} & & \multicolumn{2}{c}{$P C G_{A M G}$} \\
\cline { 3 - 4 } \cline { 6 - 7 } & $\alpha$ & $N_{i t}$ & $t_{C P U}$ & & $N_{i t}$ & $t_{C P U}$ \\
\hline \hline$\frac{1}{64}$ & $\alpha=10^{3}$ & 475 & $1.58+4.50$ & & 195 & $2.22+1.94$ \\
& $\alpha=10^{2}$ & 285 & $1.60+2.84$ & & 64 & $2.18+0.66$ \\
& $\alpha=1$ & 50 & $1.27+0.46$ & & 8 & $1.97+0.12$ \\
\hline$\frac{1}{128}$ & $\alpha=10^{3}$ & 415 & $9.63+18.63$ & & 192 & $14.73+8.84$ \\
& $\alpha=10^{2}$ & 345 & $9.51+15.32$ & & 65 & $15.03+2.93$ \\
\cline { 6 - 7 }$\frac{1}{256}$ & $\alpha=1$ & 52 & $8.14+2.19$ & & 9 & $14.39+0.43$ \\
& $\alpha=10^{3}$ & 546 & $102.33+129.59$ & & 192 & $249.91+49.38$ \\
& $\alpha=10^{2}$ & 383 & $101.38+91.70$ & & 66 & $252.64+17.03$ \\
& $\alpha=1$ & 54 & $111.51+11.36$ & & 9 & $271.42+2.14$ \\
\hline
\end{tabular}

Table 16: $L^{2}$-norms of the error for test problem $4, \alpha=1$

\begin{tabular}{ccccccc}
\hline$h$ & $\left\|u-u^{h}\right\|_{L^{2}}$ & Rate & $\left\|q_{x}-q_{x}^{h}\right\|_{L^{2}}$ & Rate & $\left\|q_{y}-q_{y}^{h}\right\|_{L^{2}}$ & Rate \\
\hline \hline$\frac{1}{16}$ & $8.06 E-03$ & - & $1.77 E-01$ & - & $1.71 E-01$ & - \\
$\frac{1}{32}$ & $3.35 E-03$ & 1.27 & $8.91 E-02$ & 0.99 & $8.55 E-02$ & 1.00 \\
$\frac{1}{64}$ & $1.63 E-03$ & 1.04 & $4.46 E-02$ & 1.00 & $4.27 E-02$ & 1.00 \\
$\frac{1}{128}$ & $8.27 E-04$ & 0.98 & $2.23 E-02$ & 1.00 & $2.13 E-02$ & 1.00 \\
$\frac{1}{256}$ & $4.19 E-04$ & 0.98 & $1.12 E-02$ & 1.00 & $1.07 E-02$ & 1.00 \\
\hline
\end{tabular}


Table 17: $L^{2}$-norms of the error for test problem $4, \alpha=10^{1}$

\begin{tabular}{ccccccc}
\hline$h$ & $\left\|u-u^{h}\right\|_{L^{2}}$ & Rate & $\left\|q_{x}-q_{x}^{h}\right\|_{L^{2}}$ & Rate & $\left\|q_{y}-q_{y}^{h}\right\|_{L^{2}}$ & Rate \\
\hline \hline$\frac{1}{16}$ & $1.34 E-02$ & - & $1.77 E+00$ & - & $1.73 E+00$ & - \\
$\frac{1}{32}$ & $4.62 E-03$ & 1.54 & $8.92 E-01$ & 0.99 & $8.67 E-01$ & 1.00 \\
$\frac{1}{64}$ & $2.22 E-03$ & 1.06 & $4.47 E-01$ & 1.00 & $4.33 E-01$ & 1.00 \\
$\frac{1}{128}$ & $1.17 E-03$ & 0.93 & $2.24 E-01$ & 1.00 & $2.17 E-01$ & 1.00 \\
$\frac{1}{256}$ & $6.05 E-04$ & 0.95 & $1.12 E-01$ & 1.00 & $1.08 E-01$ & 1.00 \\
\hline
\end{tabular}

Table 18: $L^{2}$-norms of the error for test problem $4, \alpha=10^{2}$

\begin{tabular}{ccccccc}
\hline$h$ & $\left\|u-u^{h}\right\|_{L^{2}}$ & Rate & $\left\|q_{x}-q_{x}^{h}\right\|_{L^{2}}$ & Rate & $\left\|q_{y}-q_{y}^{h}\right\|_{L^{2}}$ & Rate \\
\hline \hline$\frac{1}{16}$ & $1.17 E-01$ & - & $1.77 E+01$ & - & $1.74 E+01$ & - \\
$\frac{1}{32}$ & $2.90 E-02$ & 2.01 & $8.94 E+00$ & 0.99 & $8.69 E+00$ & 1.00 \\
$\frac{1}{64}$ & $7.14 E-03$ & 2.02 & $4.48 E+00$ & 1.00 & $4.34 E+00$ & 1.00 \\
$\frac{1}{128}$ & $1.89 E-03$ & 1.92 & $2.24 E+00$ & 1.00 & $2.17 E+00$ & 1.00 \\
$\frac{1}{256}$ & $6.60 E-04$ & 1.52 & $1.12 E+00$ & 1.00 & $1.09 E+00$ & 1.00 \\
\hline
\end{tabular}

Table 19: Iteration count and timings (set-up and solution time) for $p-M I N R E S$ and $P C G$ - Test problem 4

\begin{tabular}{ccccccc}
\hline & & \multicolumn{2}{c}{$p-M I N R E S$} & & \multicolumn{2}{c}{$P C G$} \\
\cline { 3 - 4 } \cline { 6 - 7 }$h$ & $\alpha$ & $N_{i t}$ & $t_{C P U}$ & & $N_{i t}$ & $t_{C P U}$ \\
\hline \hline$\frac{1}{64}$ & $\alpha=10^{2}$ & 65 & 1.23 & & 85 & $0.97+0.87$ \\
& $\alpha=10^{1}$ & 68 & 1.31 & & 84 & $0.95+0.85$ \\
& $\alpha=1$ & 68 & 1.32 & & 83 & $0.94+0.83$ \\
\hline$\frac{1}{128}$ & $\alpha=10^{2}$ & 65 & 8.69 & & 165 & $15.48+7.47$ \\
& $\alpha=10^{1}$ & 67 & 8.77 & & 165 & $15.27+7.62$ \\
$\frac{1}{256}$ & $\alpha=1$ & 68 & 9.26 & & 162 & $15.33+7.27$ \\
\hline & $\alpha=10^{2}$ & 64 & 44.08 & & 325 & $240.71+73.00$ \\
& $\alpha=1$ & 67 & 48.45 & & 323 & $238.17+73.01$ \\
& $\alpha=1$ & 68 & 45.95 & & 318 & $245.11+71.28$ \\
\hline
\end{tabular}


Table 20: Iteration count and timings (set-up and solution time) for $p-M I N R E S_{A M G}$ and $P C G_{A M G}$ - Test problem 4

\begin{tabular}{ccccccc}
\hline \multirow{2}{*}{$h$} & & \multicolumn{2}{c}{$p-M I N R E S_{A M G}$} & & \multicolumn{2}{c}{$P C G_{A M G}$} \\
\cline { 3 - 4 } \cline { 6 - 7 } & $\alpha$ & $N_{i t}$ & $t_{C P U}$ & & $N_{i t}$ & $t_{C P U}$ \\
\hline \hline$\frac{1}{64}$ & $\alpha=10^{2}$ & 78 & $1.33+0.75$ & & 12 & $2.12+0.16$ \\
& $\alpha=10^{1}$ & 82 & $1.38+0.78$ & & 12 & $2.08+0.16$ \\
& $\alpha=1$ & 83 & $1.35+0.74$ & & 12 & $2.12+0.16$ \\
\hline$\frac{1}{128}$ & $\alpha=10^{2}$ & 79 & $8.15+3.21$ & & 12 & $14.63+0.64$ \\
& $\alpha=10^{1}$ & 83 & $8.08+3.36$ & & 12 & $14.76+0.58$ \\
& $\alpha=1$ & 84 & $8.04+3.44$ & & 12 & $15.03+0.61$ \\
\hline$\frac{1}{256}$ & $\alpha=10^{2}$ & 78 & $102.86+20.72$ & & 13 & $278.41+3.70$ \\
& $\alpha=10^{1}$ & 85 & $108.85+21.10$ & & 13 & $252.37+3.28$ \\
& $\alpha=1$ & 85 & $114.45+21.39$ & & 12 & $260.69+2.93$ \\
\hline
\end{tabular}

Table 21: $L^{2}$-norms of the error for test problem 5

Structured Meshes

\begin{tabular}{ccccccc}
\hline$h$ & $\left\|u-u^{h}\right\|_{L^{2}}$ & Rate & $\left\|q_{x}-q_{x}^{h}\right\|_{L^{2}}$ & Rate & $\left\|q_{y}-q_{y}^{h}\right\|_{L^{2}}$ & Rate \\
\hline \hline$\frac{1}{16}$ & $6.32 E-05$ & - & $4.08 E-03$ & - & $4.08 E-03$ & - \\
$\frac{1}{32}$ & $1.59 E-05$ & 1.99 & $2.05 E-03$ & 0.99 & $2.05 E-03$ & 0.99 \\
$\frac{1}{64}$ & $3.98 E-06$ & 2.00 & $1.03 E-03$ & 1.00 & $1.03 E-03$ & 1.00 \\
$\frac{1}{128}$ & $9.97 E-07$ & 2.00 & $5.13 E-04$ & 1.00 & $5.13 E-04$ & 1.00 \\
$\frac{1}{256}$ & $2.49 E-07$ & 2.00 & $2.57 E-04$ & 1.00 & $2.57 E-04$ & 1.00 \\
\hline \multicolumn{7}{c}{ Distorted Meshes } \\
\hline$h$ & $\left\|u-u^{h}\right\|_{L^{2}}$ & Rate & $\left\|q_{x}-q_{x}^{h}\right\|_{L^{2}}$ & Rate & $\left\|q_{y}-q_{y}^{h}\right\|_{L^{2}}$ & Rate \\
\hline \hline$\frac{1}{16}$ & $6.91 E-05$ & - & $4.51 E-03$ & - & $4.90 E-03$ & - \\
$\frac{1}{32}$ & $1.78 E-05$ & 1.96 & $2.41 E-03$ & 0.90 & $2.37 E-03$ & 1.05 \\
$\frac{1}{64}$ & $4.30 E-06$ & 2.05 & $1.16 E-03$ & 1.06 & $1.16 E-03$ & 1.03 \\
$\frac{1}{128}$ & $1.06 E-06$ & 2.02 & $5.66 E-04$ & 1.03 & $5.65 E-04$ & 1.04 \\
$\frac{1}{256}$ & $2.60 E-07$ & 2.02 & $2.77 E-04$ & 1.03 & $2.77 E-04$ & 1.03 \\
\hline
\end{tabular}


Table 22: Iteration count and timings (set-up and solution time) for $p-M I N R E S$ and PCG - Test problem 5

\begin{tabular}{|c|c|c|c|c|}
\hline \multirow[b]{3}{*}{$h$} & \multicolumn{4}{|c|}{ Structured Meshes } \\
\hline & \multicolumn{2}{|c|}{$p-M I N R E S$} & \multicolumn{2}{|c|}{$P C G$} \\
\hline & $N_{i t}$ & $t_{C P U}$ & $N_{i t}$ & $t_{C P U}$ \\
\hline$\frac{1}{64}$ & 43 & 0.82 & 91 & $0.96+0.95$ \\
\hline$\frac{1}{128}$ & 43 & 5.43 & 164 & $15.01+7.83$ \\
\hline$\frac{1}{256}$ & 43 & 28.78 & 310 & $243.92+70.11$ \\
\hline \multirow[b]{3}{*}{$h$} & \multicolumn{4}{|c|}{ Distorted Meshes } \\
\hline & \multicolumn{2}{|c|}{$p-M I N R E S$} & \multicolumn{2}{|c|}{$P C G$} \\
\hline & $N_{i t}$ & $t_{C P U}$ & $N_{i t}$ & $t_{C P U}$ \\
\hline$\frac{1}{64}$ & 52 & 0.99 & 97 & $0.97+1.04$ \\
\hline$\frac{1}{128}$ & 51 & 6.56 & 190 & $15.25+9.24$ \\
\hline$\frac{1}{256}$ & 49 & 32.68 & 369 & $237.46+82.39$ \\
\hline
\end{tabular}

Table 23: Iteration count and timings (set-up and solution time) for $p-M I N R E S_{A M G}$ and $P C G_{A M G}$ - Test problem 5

\begin{tabular}{|c|c|c|c|c|}
\hline \multirow[b]{3}{*}{$h$} & \multicolumn{4}{|c|}{ Structured Meshes } \\
\hline & \multicolumn{2}{|c|}{$p-M I N R E S_{A M G}$} & \multicolumn{2}{|c|}{$P C G_{A M G}$} \\
\hline & $N_{i t}$ & $t_{C P U}$ & $N_{i t}$ & $t_{C P U}$ \\
\hline$\frac{1}{64}$ & 48 & $1.28+0.45$ & 9 & $1.97+0.15$ \\
\hline$\frac{1}{128}$ & 48 & $8.01+2.02$ & 9 & $13.65+0.46$ \\
\hline$\frac{1}{256}$ & 48 & $112.57+10.81$ & 10 & $224.68+2.63$ \\
\hline \multirow[b]{3}{*}{$h$} & \multicolumn{4}{|c|}{ Distorted Meshes } \\
\hline & \multicolumn{2}{|c|}{$p-M I N R E S_{A M G}$} & \multicolumn{2}{|c|}{$P C G_{A M G}$} \\
\hline & $N_{i t}$ & $t_{C P U}$ & $N_{i t}$ & $t_{C P U}$ \\
\hline$\frac{1}{64}$ & 63 & $1.62+0.66$ & 16 & $2.56+0.30$ \\
\hline$\frac{1}{128}$ & 61 & $9.05+3.08$ & 16 & $17.39+0.85$ \\
\hline$\frac{1}{256}$ & 63 & $112.91+15.00$ & 14 & $277.21+3.47$ \\
\hline
\end{tabular}

Table 24: $L^{2}$-norms of the error for test problem $1, \epsilon=0.9$

\begin{tabular}{ccccccc}
\hline$h$ & $\left\|u-u^{h}\right\|_{L^{2}}$ & Rate & $\left\|q_{x}-q_{x}^{h}\right\|_{L^{2}}$ & Rate & $\left\|q_{y}-q_{y}^{h}\right\|_{L^{2}}$ & Rate \\
\hline \hline$\frac{1}{16}$ & $2.94 E-03$ & - & $1.66 E-01$ & - & $8.34 E-02$ & - \\
$\frac{1}{32}$ & $6.10 E-04$ & 2.27 & $3.45 E-02$ & 2.27 & $9.47 E-03$ & 3.14 \\
$\frac{1}{64}$ & $1.49 E-04$ & 2.03 & $6.87 E-03$ & 2.33 & $2.96 E-03$ & 1.68 \\
$\frac{1}{128}$ & $3.72 E-05$ & 2.00 & $1.69 E-03$ & 2.03 & $7.29 E-04$ & 2.02 \\
$\frac{1}{256}$ & $9.31 E-06$ & 2.00 & $4.20 E-04$ & 2.01 & $1.81 E-04$ & 2.01 \\
\hline
\end{tabular}


Table 25: $L^{2}$-norms of the error for test problem $1, \epsilon=0.99$

\begin{tabular}{ccccccc}
\hline$h$ & $\left\|u-u^{h}\right\|_{L^{2}}$ & Rate & $\left\|q_{x}-q_{x}^{h}\right\|_{L^{2}}$ & Rate & $\left\|q_{y}-q_{y}^{h}\right\|_{L^{2}}$ & Rate \\
\hline \hline$\frac{1}{16}$ & $8.57 E-03$ & - & $6.42 E-01$ & - & $1.56 E+00$ & - \\
$\frac{1}{32}$ & $2.43 E-03$ & 1.82 & $4.82 E-01$ & 0.41 & $1.48 E+00$ & 0.07 \\
$\frac{1}{64}$ & $5.88 E-04$ & 2.05 & $3.14 E-01$ & 0.62 & $1.04 E+00$ & 0.50 \\
$\frac{1}{128}$ & $1.06 E-04$ & 2.47 & $1.47 E-01$ & 1.10 & $3.64 E-01$ & 1.52 \\
$\frac{1}{256}$ & $1.54 E-05$ & 2.78 & $3.77 E-02$ & 1.96 & $2.72 E-02$ & 3.74 \\
\hline
\end{tabular}

Table 26: $L^{2}$-norms of the error for test problem $1, \epsilon=0.999$

\begin{tabular}{ccccccc}
\hline$h$ & $\left\|u-u^{h}\right\|_{L^{2}}$ & Rate & $\left\|q_{x}-q_{x}^{h}\right\|_{L^{2}}$ & Rate & $\left\|q_{y}-q_{y}^{h}\right\|_{L^{2}}$ & Rate \\
\hline \hline$\frac{1}{16}$ & $9.78 E-03$ & - & $7.11 E-01$ & - & $1.87 E+00$ & - \\
$\frac{1}{32}$ & $3.18 E-03$ & 1.62 & $5.98 E-01$ & 0.25 & $2.18 E+00$ & $<0$ \\
$\frac{1}{64}$ & $1.02 E-03$ & 1.64 & $5.00 E-01$ & 0.26 & $2.48 E+00$ & $<0$ \\
$\frac{1}{128}$ & $3.22 E-04$ & 1.67 & $4.07 E-01$ & 0.29 & $2.69 E+00$ & $<0$ \\
$\frac{1}{256}$ & $9.50 E-05$ & 1.76 & $3.15 E-01$ & 0.37 & $2.67 E+00$ & 0.01 \\
\hline
\end{tabular}

Table 27: Iteration count and timings (set-up and solution time) for $p-M I N R E S$ and PCG - Test problem 1

\begin{tabular}{ccccccc}
\hline & & \multicolumn{2}{c}{$p-M I N R E S$} & & \multicolumn{2}{c}{$P C G$} \\
\cline { 3 - 4 } \cline { 6 - 7 }$h$ & $\epsilon$ & $N_{i t}$ & $t_{C P U}$ & & $N_{i t}$ & $t_{C P U}$ \\
\hline \hline$\frac{1}{64}$ & $\epsilon=0.999$ & 45 & 0.65 & & 88 & $0.55+0.62$ \\
& $\epsilon=0.99$ & 46 & 0.62 & & 89 & $0.57+0.62$ \\
& $\epsilon=0.9$ & 44 & 0.67 & & 97 & $0.56+0.70$ \\
\hline$\frac{1}{128}$ & $\epsilon=0.999$ & 45 & 3.35 & & 170 & $8.72+5.45$ \\
& $\epsilon=0.99$ & 46 & 3.31 & & 173 & $8.66+5.48$ \\
& $\epsilon=0.9$ & 39 & 2.78 & & 189 & $8.77+5.98$ \\
\hline$\frac{1}{256}$ & $\epsilon=0.999$ & 44 & 15.72 & & 336 & $132.36+50.83$ \\
& $\epsilon=0.99$ & 45 & 16.63 & & 337 & $136.88+52.07$ \\
& $\epsilon=0.9$ & 34 & 12.29 & & 371 & $135.56+57.25$ \\
\hline
\end{tabular}


Table 28: Iteration count and timings (set-up+solution time) for $p-M I N R E S_{A M G}$ and $P C G_{A M G}$ - Test problem 1

\begin{tabular}{ccccccc}
\hline & & \multicolumn{2}{c}{$p-M I N R E S_{A M G}$} & & \multicolumn{2}{c}{$P C G_{A M G}$} \\
\cline { 3 - 4 } \cline { 6 - 7 }$h$ & $\epsilon$ & $N_{i t}$ & $t_{C P U}$ & & $N_{i t}$ & $t_{C P U}$ \\
\hline \hline$\frac{1}{64}$ & $\epsilon=0.999$ & 57 & $0.71+0.33$ & & 12 & $0.19+0.12$ \\
& $\epsilon=0.99$ & 56 & $0.71+0.34$ & & 13 & $0.19+0.13$ \\
& $\epsilon=0.9$ & 55 & $0.66+0.30$ & & 12 & $0.19+0.13$ \\
\hline$\frac{1}{128}$ & $\epsilon=0.999$ & 57 & $3.18+1.05$ & & 13 & $6.55+0.43$ \\
& $\epsilon=0.99$ & 57 & $3.24+1.06$ & & 12 & $6.52+0.39$ \\
& $\epsilon=0.9$ & 57 & $3.21+1.11$ & & 13 & $6.60+0.42$ \\
\hline$\frac{1}{256}$ & $\epsilon=0.999$ & 59 & $25.11+6.67$ & & 13 & $99.00+2.09$ \\
& $\epsilon=0.99$ & 61 & $25.21+7.11$ & & 13 & $99.13+2.09$ \\
& $\epsilon=0.9$ & 57 & $25.25+6.62$ & & 13 & $98.75+2.10$ \\
\hline
\end{tabular}

Table 29: $L^{2}$-norms of the error for test problem 2, $\alpha=10^{-2}$

\begin{tabular}{cccccccc}
\hline$h$ & $\left\|u-u^{h}\right\|_{L^{2}}$ & Rate & $\left\|q_{x}-q_{x}^{h}\right\|_{L^{2}}$ & Rate & $\left\|q_{y}-q_{y}^{h}\right\|_{L^{2}}$ & Rate & $u_{\min }$ \\
\hline \hline$\frac{1}{16}$ & $1.00 E-04$ & - & $1.17 E-04$ & - & $2.40 E-04$ & - & $8.28 E-04$ \\
$\frac{1}{32}$ & $2.55 E-05$ & 1.97 & $2.96 E-05$ & 1.99 & $6.03 E-05$ & 1.99 & $2.02 E-04$ \\
$\frac{1}{64}$ & $6.39 E-06$ & 1.99 & $7.42 E-06$ & 2.00 & $1.51 E-05$ & 2.00 & $4.95 E-05$ \\
$\frac{1}{128}$ & $1.60 E-06$ & 2.00 & $1.86 E-06$ & 2.00 & $3.78 E-06$ & 2.00 & $1.22 E-05$ \\
$\frac{1}{256}$ & $4.00 E-07$ & 2.00 & $4.64 E-07$ & 2.00 & $9.45 E-07$ & 2.00 & $3.03 E-06$ \\
\hline
\end{tabular}

Table 30: $L^{2}$-norms of the error for test problem 2, $\alpha=1$

\begin{tabular}{cccccccc}
\hline$h$ & $\left\|u-u^{h}\right\|_{L^{2}}$ & Rate & $\left\|q_{x}-q_{x}^{h}\right\|_{L^{2}}$ & Rate & $\left\|q_{y}-q_{y}^{h}\right\|_{L^{2}}$ & Rate & $u_{\min }$ \\
\hline \hline$\frac{1}{16}$ & $1.46 E-04$ & - & $2.98 E-04$ & - & $2.98 E-04$ & - & $7.69 E-04$ \\
$\frac{1}{32}$ & $3.70 E-05$ & 1.98 & $7.50 E-05$ & 1.99 & $7.50 E-05$ & 1.99 & $1.82 E-04$ \\
$\frac{1}{64}$ & $9.29 E-06$ & 1.99 & $1.88 E-05$ & 2.00 & $1.88 E-05$ & 2.00 & $4.37 E-05$ \\
$\frac{1}{128}$ & $2.32 E-06$ & 2.00 & $4.70 E-06$ & 2.00 & $4.70 E-06$ & 2.00 & $1.06 E-05$ \\
$\frac{1}{256}$ & $5.81 E-07$ & 2.00 & $1.17 E-06$ & 2.00 & $1.17 E-06$ & 2.00 & $2.60 E-06$ \\
\hline
\end{tabular}

Table 31: $L^{2}$-norms of the error for test problem 2, $\alpha=10^{2}$

\begin{tabular}{cccccccc}
\hline$h$ & $\left\|u-u^{h}\right\|_{L^{2}}$ & Rate & $\left\|q_{x}-q_{x}^{h}\right\|_{L^{2}}$ & Rate & $\left\|q_{y}-q_{y}^{h}\right\|_{L^{2}}$ & Rate & $u_{\min }$ \\
\hline \hline$\frac{1}{16}$ & $6.08 E-04$ & - & $4.91 E-02$ & - & $1.07 E-03$ & - & $5.41 E-04$ \\
$\frac{1}{32}$ & $1.56 E-04$ & 1.97 & $1.24 E-02$ & 1.98 & $2.72 E-04$ & 1.98 & $1.17 E-04$ \\
$\frac{1}{64}$ & $3.88 E-05$ & 2.00 & $3.11 E-03$ & 2.00 & $6.82 E-05$ & 2.00 & $2.58 E-05$ \\
$\frac{1}{128}$ & $9.66 E-06$ & 2.00 & $7.79 E-04$ & 2.00 & $1.71 E-05$ & 2.00 & $5.86 E-06$ \\
$\frac{1}{256}$ & $2.41 E-06$ & 2.00 & $1.95 E-04$ & 2.00 & $4.27 E-06$ & 2.00 & $1.37 E-06$ \\
\hline
\end{tabular}


Table 32: Iteration count and timings (set-up and solution time) for $p-M I N R E S$ and PCG - Test problem 2

\begin{tabular}{ccccccc}
\hline \multirow{2}{*}{$h$} & \multirow{2}{*}{$\alpha$} & \multicolumn{2}{c}{$p-M I N R E S$} & & \multicolumn{2}{c}{$P C G$} \\
\cline { 3 - 4 } \cline { 6 - 6 }$N_{i t}$ & $t_{C P U}$ & & $N_{i t}$ & $t_{C P U}$ \\
\hline \hline$\frac{1}{64}$ & $\alpha=10^{2}$ & 38 & 0.65 & & 68 & $0.57+0.49$ \\
& $\alpha=1$ & 36 & 0.58 & & 81 & $0.59+0.57$ \\
& $\alpha=10^{-2}$ & 37 & 0.58 & & 83 & $0.56+0.59$ \\
\hline$\frac{1}{128}$ & $\alpha=10^{2}$ & 33 & 2.54 & & 136 & $8.82+4.35$ \\
& $\alpha=1$ & 33 & 2.53 & & 158 & $8.66+4.99$ \\
& $\alpha=10^{-2}$ & 33 & 2.56 & & 170 & $8.65+5.35$ \\
\hline$\frac{1}{256}$ & $\alpha=10^{2}$ & 29 & 10.51 & & 274 & $133.96+42.05$ \\
& $\alpha=1$ & 29 & 10.51 & & 311 & $135.29+47.04$ \\
& $\alpha=10^{-2}$ & 30 & 10.78 & & 353 & $139.34+54.00$ \\
\hline
\end{tabular}

Table 33: Iteration count and timings (set-up and solution time) for $p-M I N R E S_{A M G}$ and $P C G_{A M G}$ - Test problem 2

\begin{tabular}{ccccccc}
\hline \multirow{2}{*}{$h$} & $\alpha$ & \multicolumn{2}{c}{$p-M I N R E S_{A M G}$} & & \multicolumn{2}{c}{$P C G_{A M G}$} \\
\cline { 3 - 4 } \cline { 6 - 7 } & $\alpha$ & $N_{i t}$ & $t_{C P U}$ & & $N_{i t}$ & $t_{C P U}$ \\
\hline \hline$\frac{1}{64}$ & $\alpha=10^{2}$ & 52 & $0.77+0.35$ & & 12 & $1.27+0.13$ \\
& $\alpha=1$ & 47 & $0.67+0.28$ & & 15 & $1.23+0.14$ \\
& $\alpha=10^{-2}$ & 54 & $0.73+0.33$ & & 19 & $1.24+0.17$ \\
\hline$\frac{1}{128}$ & $\alpha=10^{2}$ & 56 & $3.73+1.23$ & & 13 & $6.15+0.54$ \\
& $\alpha=1$ & 46 & $3.16+0.87$ & & 15 & $6.66+0.49$ \\
& $\alpha=10^{-2}$ & 53 & $3.36+1.04$ & & 20 & $6.62+0.62$ \\
\hline$\frac{1}{256}$ & $\alpha=10^{2}$ & 59 & $28.74+7.25$ & & 14 & $87.01+2.62$ \\
& $\alpha=1$ & 46 & $25.61+5.34$ & & 16 & $100.19+2.74$ \\
& $\alpha=10^{-2}$ & 56 & $26.48+6.30$ & & 21 & $95.46+3.37$ \\
\hline
\end{tabular}



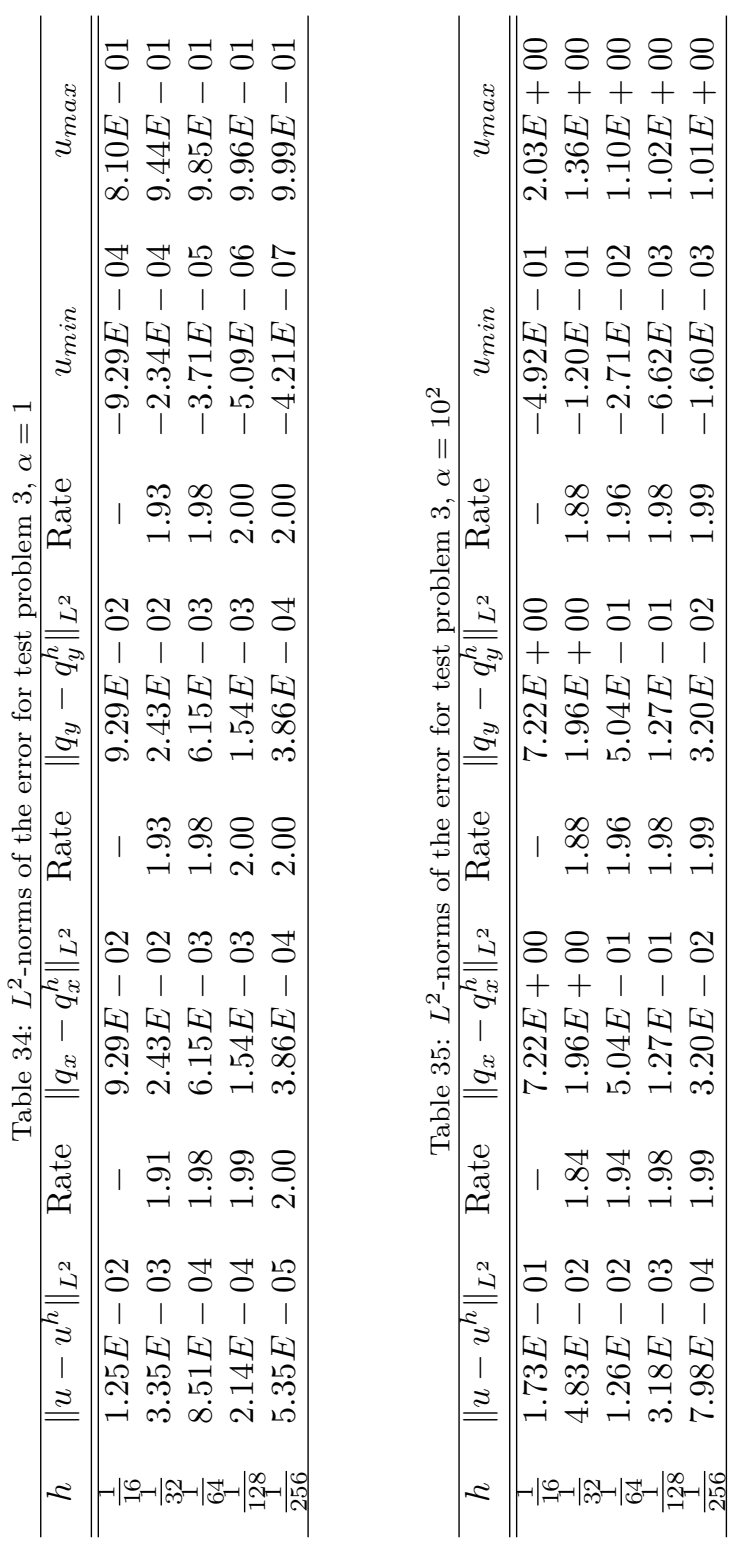


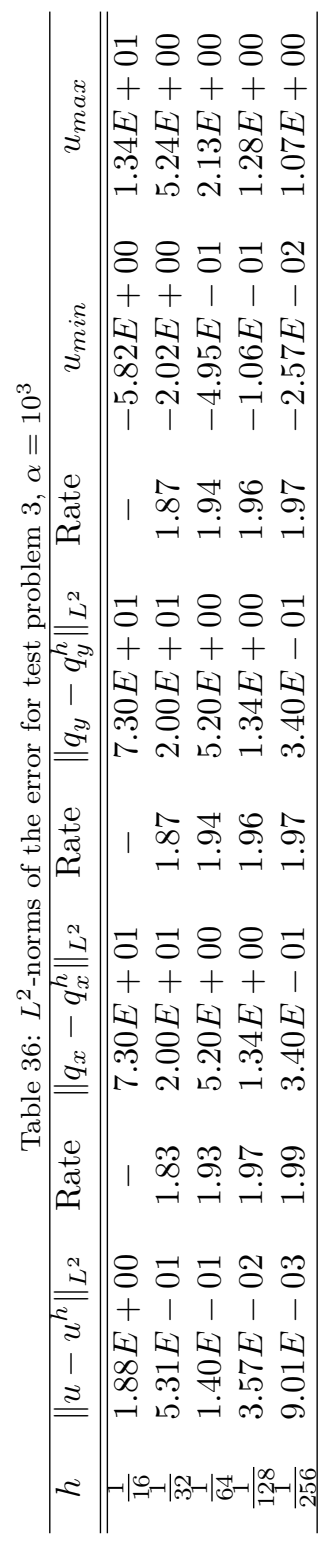


Table 37: Iteration count and timings (set-up and solution time) for $p-M I N R E S$ and PCG - Test problem 3

\begin{tabular}{ccccccc}
\hline \multirow{2}{nnnnnyy}{$h$} & & \multicolumn{2}{c}{$p-M I N R E S$} & & \multicolumn{2}{c}{$P C G$} \\
\cline { 3 - 4 } \cline { 6 - 7 } & $\alpha$ & $N_{i t}$ & $t_{C P U}$ & & $N_{i t}$ & $t_{C P U}$ \\
\hline \hline$\frac{1}{64}$ & $\alpha=10^{3}$ & 351 & 5.07 & & 12 & $0.57+0.13$ \\
& $\alpha=10^{2}$ & 254 & 3.81 & & 22 & $0.55+0.18$ \\
& $\alpha=1$ & 22 & 0.33 & & 79 & $0.54+0.55$ \\
\hline$\frac{1}{128}$ & $\alpha=10^{3}$ & 466 & 38.08 & & 14 & $8.63+0.50$ \\
& $\alpha=10^{2}$ & 283 & 21.82 & & 40 & $8.64+1.29$ \\
& $\alpha=1$ & 18 & 1.37 & & 154 & $8.58+4.77$ \\
\hline$\frac{1}{256}$ & $\alpha=10^{3}$ & 554 & 204.61 & & 20 & $133.07+3.06$ \\
& $\alpha=10^{2}$ & 300 & 111.65 & & 77 & $134.12+11.60$ \\
& $\alpha=1$ & 13 & 4.76 & & 306 & $134.98+45.43$ \\
\hline
\end{tabular}

Table 38: Iteration count and timings (set-up and solution time) for $p-M I N R E S_{A M G}$ and $P C G_{A M G}$ - Test problem 3

\begin{tabular}{ccccccc}
\hline & & \multicolumn{2}{c}{$p-M I N R E S_{A M G}$} & & \multicolumn{2}{c}{$P C G_{A M G}$} \\
\cline { 3 - 4 } \cline { 6 - 7 }$h$ & $\alpha$ & $N_{i t}$ & $t_{C P U}$ & & $N_{i t}$ & $t_{C P U}$ \\
\hline \hline$\frac{1}{64}$ & $\alpha=10^{3}$ & 369 & $0.73+2.19$ & & 120 & $2.52+1.25$ \\
& $\alpha=10^{2}$ & 267 & $0.77+1.60$ & & 39 & $2.47+0.42$ \\
& $\alpha=1$ & 45 & $0.66+0.26$ & & 14 & $1.20+0.14$ \\
\hline$\frac{1}{128}$ & $\alpha=10^{3}$ & 495 & $3.52+11.27$ & & 136 & $13.61+7.66$ \\
& $\alpha=10^{2}$ & 300 & $3.60+6.93$ & & 51 & $13.62+2.87$ \\
& $\alpha=1$ & 42 & $3.11+0.80$ & & 15 & $6.60+0.46$ \\
\hline$\frac{1}{256}$ & $\alpha=10^{3}$ & 601 & $27.29+75.95$ & & 207 & $130.16+58.57$ \\
& $\alpha=10^{2}$ & 323 & $27.35+39.92$ & & 96 & $131.03+27.39$ \\
& $\alpha=1$ & 42 & $25.46+4.79$ & & 16 & $97.42+2.46$ \\
\hline
\end{tabular}

Table 39: $L^{2}$-norms of the error for test problem $4, \alpha=1$

\begin{tabular}{ccccccc}
\hline$h$ & $\left\|u-u^{h}\right\|_{L^{2}}$ & Rate & $\left\|q_{x}-q_{x}^{h}\right\|_{L^{2}}$ & Rate & $\left\|q_{y}-q_{y}^{h}\right\|_{L^{2}}$ & Rate \\
\hline \hline$\frac{1}{16}$ & $9.28 E-03$ & - & $3.19 E-02$ & - & $2.81 E-02$ & - \\
$\frac{1}{32}$ & $4.88 E-03$ & 0.93 & $1.67 E-02$ & 0.94 & $1.43 E-02$ & 0.97 \\
$\frac{1}{64}$ & $2.50 E-03$ & 0.96 & $8.55 E-03$ & 0.96 & $7.26 E-03$ & 0.98 \\
$\frac{1}{128}$ & $1.27 E-03$ & 0.98 & $4.33 E-03$ & 0.98 & $3.66 E-03$ & 0.99 \\
$\frac{1}{256}$ & $6.37 E-04$ & 0.99 & $2.18 E-03$ & 0.99 & $1.83 E-03$ & 0.99 \\
\hline
\end{tabular}


Table 40: $L^{2}$-norms of the error for test problem $4, \alpha=10^{1}$

\begin{tabular}{ccccccc}
\hline$h$ & $\left\|u-u^{h}\right\|_{L^{2}}$ & Rate & $\left\|q_{x}-q_{x}^{h}\right\|_{L^{2}}$ & Rate & $\left\|q_{y}-q_{y}^{h}\right\|_{L^{2}}$ & Rate \\
\hline \hline$\frac{1}{16}$ & $1.67 E-02$ & - & $4.06 E-01$ & - & $3.53 E-01$ & - \\
$\frac{1}{32}$ & $7.76 E-03$ & 1.10 & $2.14 E-01$ & 0.93 & $1.81 E-01$ & 0.96 \\
$\frac{1}{64}$ & $3.81 E-03$ & 1.03 & $1.10 E-01$ & 0.96 & $9.20 E-02$ & 0.98 \\
$\frac{1}{128}$ & $1.90 E-03$ & 1.01 & $5.57 E-02$ & 0.98 & $4.64 E-02$ & 0.99 \\
$\frac{1}{256}$ & $9.49 E-04$ & 1.00 & $2.81 E-02$ & 0.99 & $2.33 E-02$ & 0.99 \\
\hline
\end{tabular}

Table 41: $L^{2}$-norms of the error for test problem $4, \alpha=10^{2}$

\begin{tabular}{ccccccc}
\hline$h$ & $\left\|u-u^{h}\right\|_{L^{2}}$ & Rate & $\left\|q_{x}-q_{x}^{h}\right\|_{L^{2}}$ & Rate & $\left\|q_{y}-q_{y}^{h}\right\|_{L^{2}}$ & Rate \\
\hline \hline$\frac{1}{16}$ & $8.18 E-02$ & - & $4.25 E+00$ & - & $3.67 E+00$ & - \\
$\frac{1}{32}$ & $2.23 E-02$ & 1.88 & $2.24 E+00$ & 0.92 & $1.89 E+00$ & 0.96 \\
$\frac{1}{64}$ & $6.82 E-03$ & 1.71 & $1.15 E+00$ & 0.96 & $9.61 E-01$ & 0.98 \\
$\frac{1}{128}$ & $2.52 E-03$ & 1.44 & $5.84 E-01$ & 0.98 & $4.84 E-01$ & 0.99 \\
$\frac{1}{256}$ & $1.09 E-03$ & 1.20 & $2.94 E-01$ & 0.99 & $2.43 E-01$ & 0.99 \\
\hline
\end{tabular}

Table 42: Iteration count and timings (set-up and solution time) for $p-M I N R E S$ and PCG - Test problem 4

\begin{tabular}{ccccccc}
\hline & & \multicolumn{2}{c}{$p-M I N R E S$} & & \multicolumn{2}{c}{$P C G$} \\
\cline { 3 - 4 } \cline { 6 - 7 }$h$ & $\alpha$ & $N_{i t}$ & $t_{C P U}$ & & $N_{i t}$ & $t_{C P U}$ \\
\hline \hline$\frac{1}{64}$ & $\alpha=10^{2}$ & 64 & 0.83 & & 81 & $0.60+0.60$ \\
& $\alpha=10^{1}$ & 66 & 0.94 & & 80 & $0.58+0.57$ \\
& $\alpha=1$ & 66 & 0.92 & & 81 & $0.56+0.59$ \\
\hline$\frac{1}{128}$ & $\alpha=10^{2}$ & 64 & 4.64 & & 162 & $8.60+5.26$ \\
& $\alpha=10^{1}$ & 66 & 4.72 & & 162 & $8.61+5.24$ \\
& $\alpha=1$ & 66 & 4.74 & & 163 & $8.61+5.32$ \\
\hline$\frac{1}{256}$ & $\alpha=10^{2}$ & 63 & 23.41 & & 328 & $137.32+51.22$ \\
& $\alpha=10^{1}$ & 64 & 23.48 & & 326 & $135.34+50.45$ \\
& $\alpha=1$ & 66 & 24.71 & & 325 & $134.91+50.29$ \\
\hline
\end{tabular}


Table 43: Iteration count and timings (set-up and solution time) for $p-M I N R E S_{A M G}$ and $P C G_{A M G}$ - Test problem 4

\begin{tabular}{ccccccc}
\hline & & \multicolumn{2}{c}{$p-M I N R E S_{A M G}$} & & \multicolumn{2}{c}{$P C G_{A M G}$} \\
\cline { 3 - 4 } \cline { 6 - 7 }$h$ & $\alpha$ & $N_{i t}$ & $t_{C P U}$ & & $N_{i t}$ & $t_{C P U}$ \\
\hline \hline$\frac{1}{64}$ & $\alpha=10^{2}$ & 70 & $0.71+0.39$ & & 15 & $1.73+0.17$ \\
& $\alpha=10^{1}$ & 71 & $0.71+0.41$ & & 15 & $1.73+0.17$ \\
& $\alpha=1$ & 71 & $0.69+0.42$ & & 15 & $1.73+0.17$ \\
\hline$\frac{1}{128}$ & $\alpha=10^{2}$ & 70 & $3.31+1.41$ & & 16 & $9.48+0.66$ \\
& $\alpha=10^{1}$ & 72 & $3.34+1.50$ & & 16 & $9.37+0.64$ \\
$\frac{1}{256}$ & $\alpha=1$ & 71 & $3.18+1.47$ & & 15 & $9.23+0.60$ \\
& $\alpha=10^{2}$ & 70 & $25.50+8.11$ & & 17 & $115.07+3.28$ \\
& $\alpha=10^{1}$ & 73 & $26.26+8.98$ & & 16 & $115.35+3.10$ \\
& $\alpha=1$ & 73 & $26.22+8.54$ & & 16 & $115.63+3.13$ \\
\hline
\end{tabular}

Table 44: $L^{2}$-norms of the error for test problem 5

Structured Meshes

\begin{tabular}{ccccccc}
\hline$h$ & $\left\|u-u^{h}\right\|_{L^{2}}$ & Rate & $\left\|q_{x}-q_{x}^{h}\right\|_{L^{2}}$ & Rate & $\left\|q_{y}-q_{y}^{h}\right\|_{L^{2}}$ & Rate \\
\hline \hline$\frac{1}{16}$ & $8.07 E-05$ & - & $2.59 E-04$ & - & $2.59 E-04$ & - \\
$\frac{1}{32}$ & $2.02 E-05$ & 2.00 & $6.47 E-05$ & 2.00 & $6.47 E-05$ & 2.00 \\
$\frac{1}{64}$ & $5.04 E-06$ & 2.00 & $1.62 E-05$ & 2.00 & $1.62 E-05$ & 2.00 \\
$\frac{1}{128}$ & $1.26 E-06$ & 2.00 & $4.05 E-06$ & 2.00 & $4.05 E-06$ & 2.00 \\
$\frac{1}{256}$ & $3.15 E-07$ & 2.00 & $1.01 E-06$ & 2.00 & $1.01 E-06$ & 2.00 \\
\hline \multicolumn{7}{c}{ Distorted Meshes } \\
\hline$h$ & $\left\|u-u^{h}\right\|_{L^{2}}$ & Rate & $\left\|q_{x}-q_{x}^{h}\right\|_{L^{2}}$ & Rate & $\left\|q_{y}-q_{y}^{h}\right\|_{L^{2}}$ & Rate \\
\hline \hline$\frac{1}{16}$ & $8.54 E-05$ & - & $1.93 E-03$ & - & $2.03 E-03$ & - \\
$\frac{1}{32}$ & $1.98 E-05$ & 2.11 & $9.70 E-04$ & 0.99 & $9.56 E-04$ & 1.09 \\
$\frac{1}{64}$ & $5.33 E-06$ & 1.89 & $4.09 E-04$ & 1.25 & $4.06 E-04$ & 1.23 \\
$\frac{1}{128}$ & $1.30 E-06$ & 2.04 & $1.81 E-04$ & 1.18 & $1.77 E-04$ & 1.20 \\
$\frac{1}{256}$ & $3.33 E-07$ & 1.96 & $7.85 E-05$ & 1.21 & $7.85 E-05$ & 1.17 \\
\hline
\end{tabular}


Table 45: Iteration count and timings (set-up and solution time) for $p-M I N R E S$ and $P C G$ - Test problem 5

\begin{tabular}{cccccc}
\hline & \multicolumn{4}{c}{ Structured Meshes } \\
\cline { 2 - 3 }$h$ & $p-M I N R E S$ & & \multicolumn{2}{c}{$P C G$} \\
\cline { 2 - 3 } \cline { 5 - 6 } & $N_{i t}$ & $t_{C P U}$ & & $N_{i t}$ & $t_{C P U}$ \\
\hline \hline$\frac{1}{64}$ & 26 & 0.33 & & 71 & $0.58+0.52$ \\
$\frac{1}{128}$ & 23 & 1.77 & & 133 & $8.71+4.29$ \\
$\frac{1}{256}$ & 20 & 7.24 & & 251 & $137.05+39.89$ \\
\hline & \multicolumn{3}{c}{ Distorted Meshes } \\
\cline { 2 - 3 }$h$ & $p-M I N R E S$ & & \multicolumn{3}{c}{$P C G$} \\
\cline { 2 - 3 } \cline { 5 - 6 } & $N_{i t}$ & $t_{C P U}$ & & $N_{i t}$ & $t_{C P U}$ \\
\hline \hline$\frac{1}{64}$ & 43 & 0.54 & & 81 & $0.56+0.59$ \\
$\frac{1}{128}$ & 42 & 3.12 & & 157 & $8.75+5.19$ \\
$\frac{1}{256}$ & 40 & 14.76 & & 279 & $136.51+43.62$ \\
\hline
\end{tabular}

Table 46: Iteration count and timings (set-up and solution time) for $p-M I N R E S_{A M G}$ and $P C G_{A M G}$ - Test problem 5

\begin{tabular}{|c|c|c|c|c|}
\hline \multirow[b]{3}{*}{$h$} & \multicolumn{4}{|c|}{ Structured Meshes } \\
\hline & \multicolumn{2}{|c|}{$p-M I N R E S_{A M G}$} & \multicolumn{2}{|c|}{$P C G_{A M G}$} \\
\hline & $N_{i t}$ & $t_{C P U}$ & $N_{i t}$ & $t_{C P U}$ \\
\hline$\frac{1}{64}$ & 36 & $0.68+0.27$ & 14 & $1.26+0.14$ \\
\hline$\frac{1}{128}$ & 36 & $3.14+0.75$ & 15 & $6.93+0.55$ \\
\hline$\frac{1}{256}$ & 36 & $25.05+4.00$ & 15 & $96.13+2.56$ \\
\hline \multirow[b]{3}{*}{$h$} & \multicolumn{4}{|c|}{ Distorted Meshes } \\
\hline & \multicolumn{2}{|c|}{$p-M I N R E S_{A M G}$} & \multicolumn{2}{|c|}{$P C G_{A M G}$} \\
\hline & $N_{i t}$ & $t_{C P U}$ & $\overline{N_{i t}}$ & $t_{C P U}$ \\
\hline$\frac{1}{64}$ & 48 & $0.70+0.35$ & 15 & $2.41+0.17$ \\
\hline$\frac{1}{128}$ & 48 & $3.16+1.15$ & 16 & $11.74+0.75$ \\
\hline$\frac{1}{256}$ & 48 & $25.18+5.64$ & 18 & $118.08+3.41$ \\
\hline
\end{tabular}




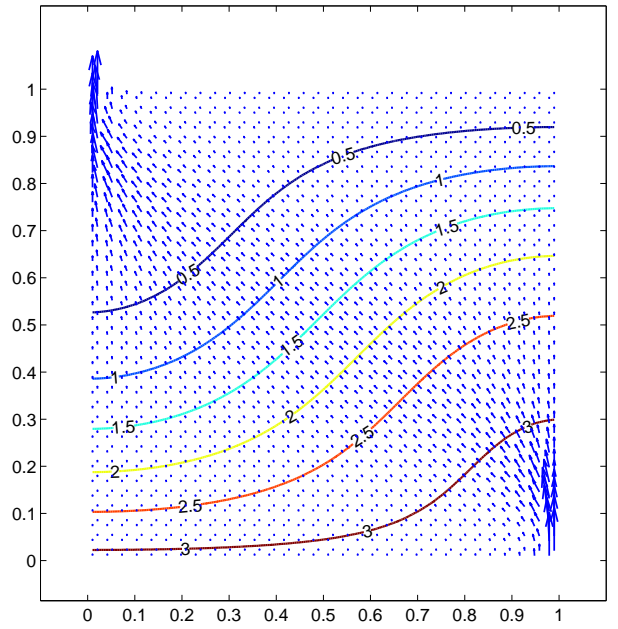

(a) Approximations for $u$ and $\mathbf{q}$

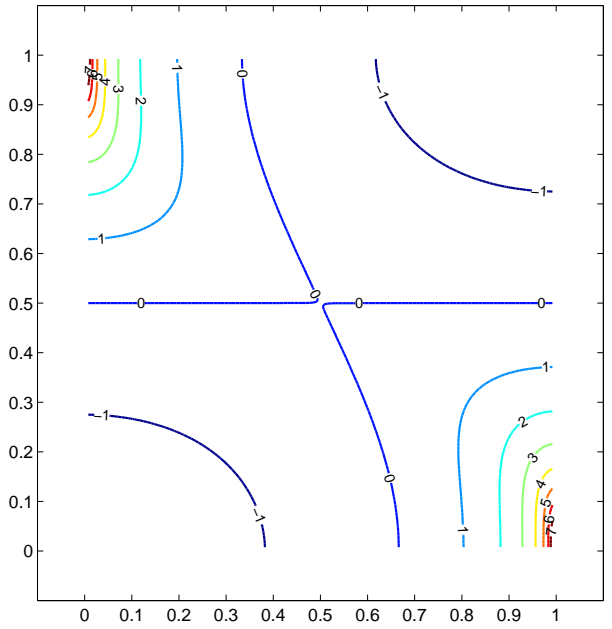

(b) Log of conductivity field $\mathcal{C}(\mathbf{x})$

Figure 1: Numerical approximations and conductivity field for $\epsilon=0.9$ - Test problem 1 


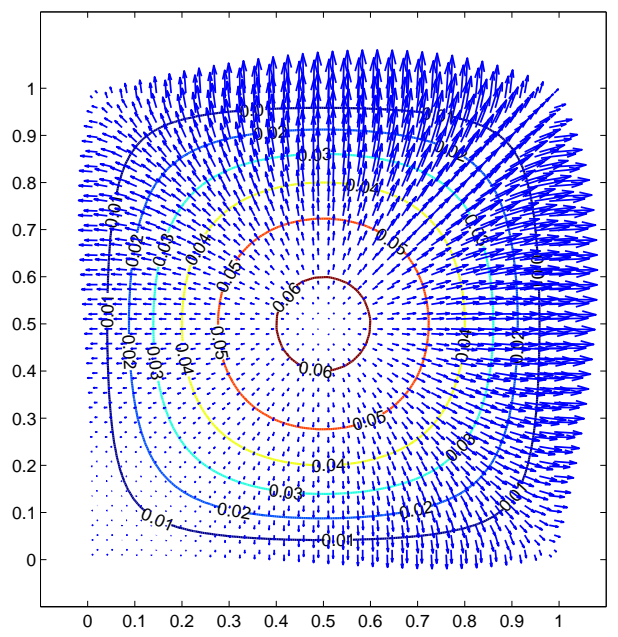

(a) Approximations for $u$ and $\mathbf{q}$

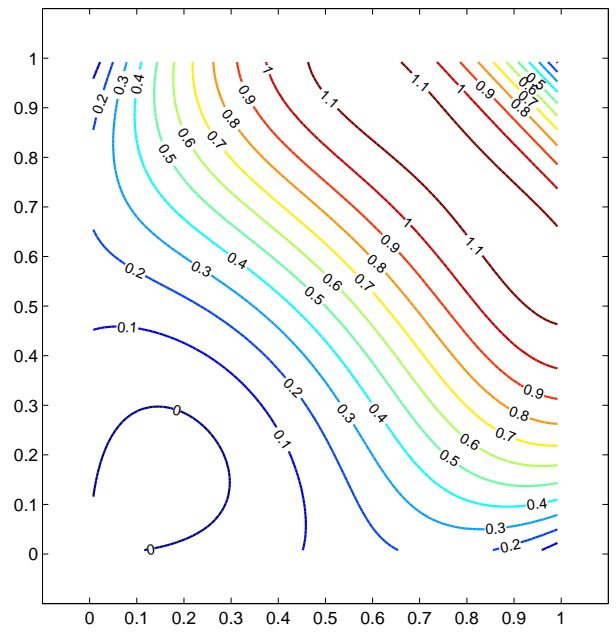

(b) Source term $f$

Figure 2: MFEM approximations and source term - Test problem 2 


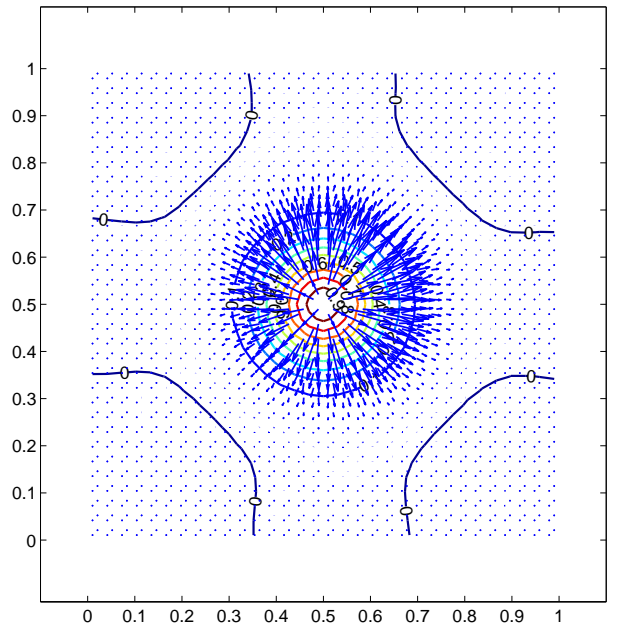

(a) Approximations for $u$ and $\mathbf{q}$

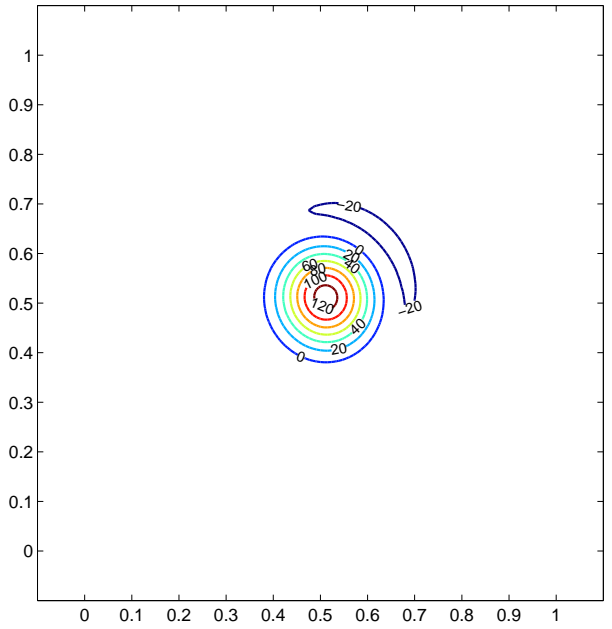

(b) Source term $f(\mathbf{x})$

Figure 3: MFEM approximations and source term for $\alpha=1$ - Test problem 3 


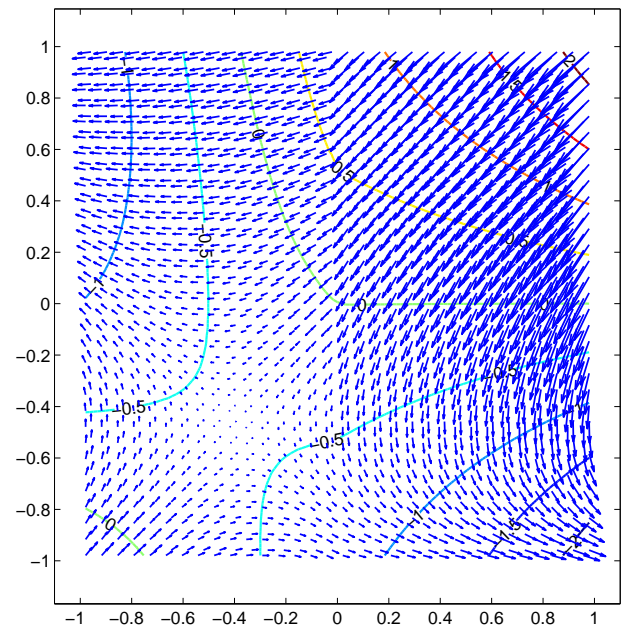

(a) $\alpha=1$

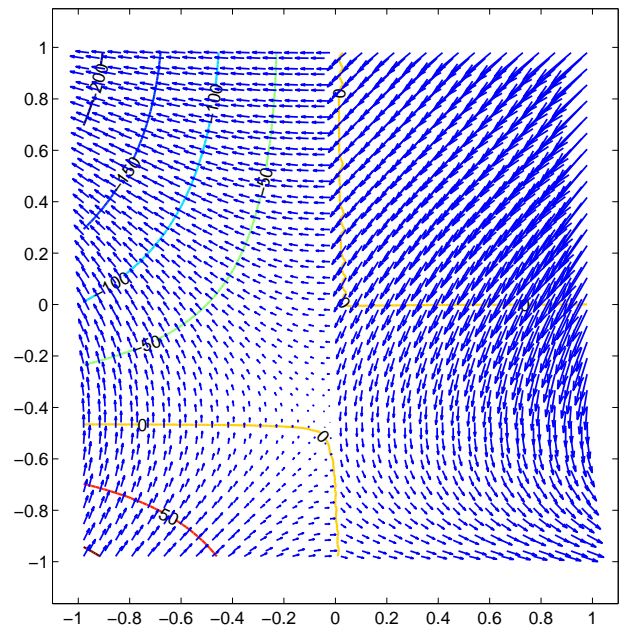

(b) $\alpha=10^{2}$

Figure 4: MFEM approximations for $\alpha=1,10^{2}$ - Test problem 4 


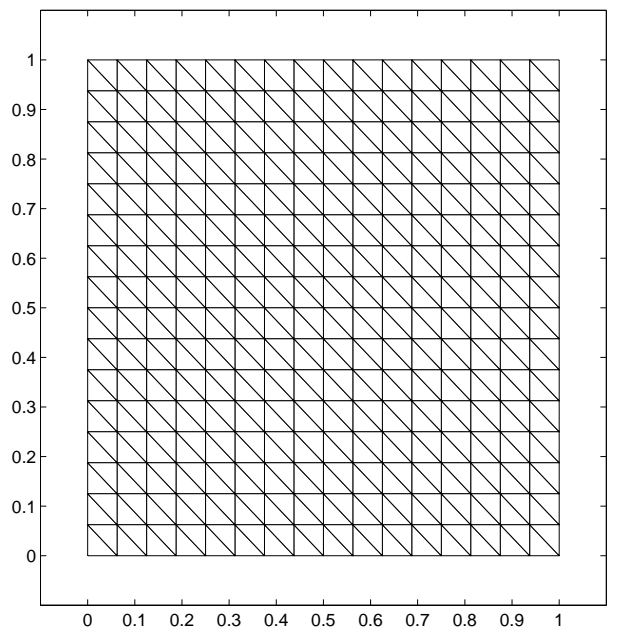

(a) Structured mesh

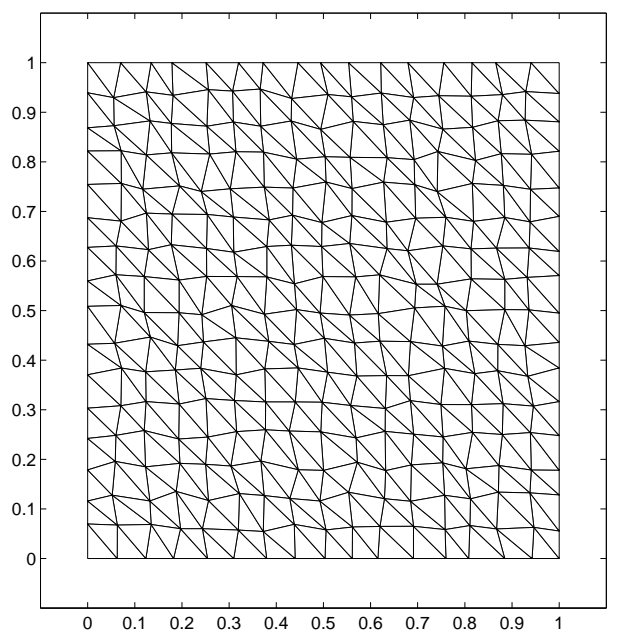

(b) Distorted mesh

Figure 5: Structured and perturbed triangular finite element meshes for $h=\frac{1}{16}$ - Test problem 5 